Florida International University FIU Digital Commons

$4-1-2011$

\title{
Space-Time Coding for Polynomial Phase Modulated Signals
}

Omar D. Granados

Florida International University, ogran002@fiu.edu

DOI: $10.25148 /$ etd.FI1 1050305

Follow this and additional works at: https://digitalcommons.fiu.edu/etd

\section{Recommended Citation}

Granados, Omar D., "Space-Time Coding for Polynomial Phase Modulated Signals" (2011). FIU Electronic Theses and Dissertations. 378.

https://digitalcommons.fiu.edu/etd/378

This work is brought to you for free and open access by the University Graduate School at FIU Digital Commons. It has been accepted for inclusion in FIU Electronic Theses and Dissertations by an authorized administrator of FIU Digital Commons. For more information, please contact dcc@fiu.edu. 


\title{
FLORIDA INTERNATIONAL UNIVERSITY
}

Miami, Florida

SPACE TIME CODING FOR POLYNOMIAL PHASE MODULATED SIGNALS

\author{
A dissertation submitted in partial fulfillment of the \\ requirements for the degree of \\ DOCTOR OF PHILOSOPHY \\ in \\ ELECTRICAL ENGINEERING \\ by \\ Omar Granados
}

2011 


\section{To: Dean Amir Mirmiran}

College of Engineering and Computing

This dissertation, written by Omar Granados, and entitled Space-Time Coding for Polynomial Phase Modulated Signals, having been approved in respect to style and intellectual content, is referred to you for judgment.

We have read this dissertation and recommend that it be approved.

$\begin{array}{r}\text { Kang K. Yen } \\ \hline \text { Hao Zhu } \\ \hline \text { Shih-Ming Lee } \\ \hline \text { Jean H. Andrian, Major Professor }\end{array}$

Date of Defense: April 1, 2011

The dissertation of Omar Granados is approved.

Dean Amir Mirmiran
College of Engineering and Computing

\begin{tabular}{r}
\hline Interim Dean Kevin O’Shea \\
University Graduate School
\end{tabular}

Florida International University, 2011 
DEDICATION

To My Family 


\section{ACKNOWLEDGMENTS}

First of all, I would like to express my sincere gratitude to Dr. Jean Andrian for his constant support and guidance. He provided me with encouragement and an interesting working environment that allowed me to finish this dissertation.

Special thanks go to the members of my dissertation committee: Dr. Kang Yen whose constant support was essential for me to complete the Ph.D. program, Dr. Hao Zhu, and Dr. Shih-Ming Lee for their advice and teachings during the course of my career. In addition, I would also like to acknowledge Dr. Chen Liu for his support and understanding during my last semester.

I am also indebted to the past and current members of the wireless communications research laboratory for all of our insightful technical conversations and for making my time as a Ph.D. student an enjoyable one. Also thanks are due to the administrative staff at the Department of Electrical and Computer Engineering for their helpful and friendly help and support.

Lastly, I want thank my family for all their hard work and want to recognize them as a major source of inspiration and encouragement for pursuing higher education. 


\section{ABSTRACT OF THE DISSERTATION}

SPACE-TIME CODING FOR POLYNOMIAL PHASE MODULATED SIGNALS

by

Omar Granados

Florida International University, 2011

Miami, Florida

\section{Professor Jean H. Andrian, Major Professor}

Polynomial phase modulated (PPM) signals have been shown to provide improved error rate performance with respect to conventional modulation formats under additive white Gaussian noise and fading channels in single-input single-output (SISO) communication systems. In this dissertation, systems with two and four transmit antennas using PPM signals were presented. In both cases we employed full-rate space-time block codes in order to take advantage of the multipath channel. For two transmit antennas, we used the orthogonal space-time block code (OSTBC) proposed by Alamouti and performed symbol-wise decoding by estimating the phase coefficients of the PPM signal using three different methods: maximum-likelihood (ML), sub-optimal ML (S-ML) and the highorder ambiguity function (HAF). In the case of four transmit antennas, we used the fullrate quasi-OSTBC (QOSTBC) proposed by Jafarkhani. However, in order to ensure the best error rate performance, PPM signals were selected such as to maximize the QOSTBC's minimum coding gain distance (CGD). Since this method does not always provide a unique solution, an additional criterion known as maximum channel interference coefficient (CIC) was proposed. Through Monte Carlo simulations it was shown that by using QOSTBCs along with the properly selected PPM constellations 
based on the CGD and CIC criteria, full diversity in flat fading channels and thus, low BER at high signal-to-noise ratios (SNR) can be ensured. Lastly, the performance of symbol-wise decoding for QOSTBCs was evaluated. In this case a quasi zero-forcing method was used to decouple the received signal and it was shown that although this technique reduces the decoding complexity of the system, there is a penalty to be paid in terms of error rate performance at high SNRs. 


\section{TABLE OF CONTENTS}

CHAPTER

PAGE

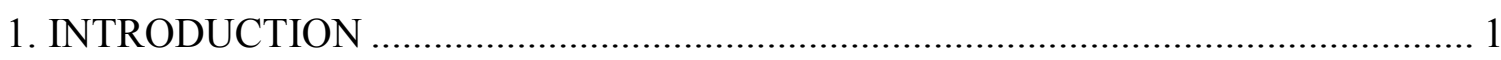

1.1 Objective and Contributions......................................................................... 3

2. POLYNOMIAL PHASE MODULATION .............................................................. 7

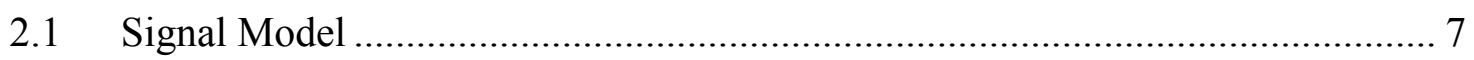

2.2 Gray Bit Labeling for Polynomial Phase Modulated Signals ........................... 8

2.3 Optimum PPM Demodulator using Maximum-Likelihood Estimation............. 10

2.4 Suboptimal Maximum-Likelihood Demodulation ........................................... 12

2.5 Parameter Estimation Using the High-Order Ambiguity Function.................... 14

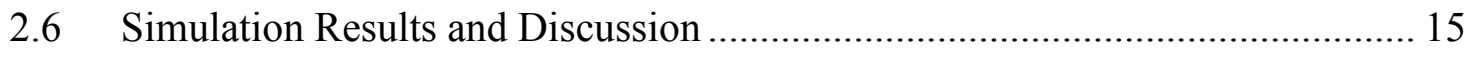

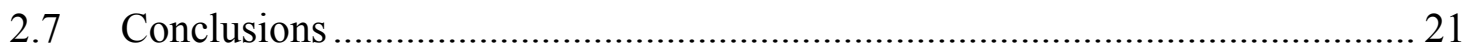

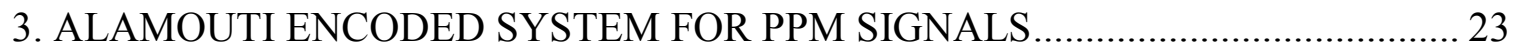

3.1 Multiplexing and Diversity in MIMO Systems........................................... 23

3.2 System Model for 2x1 Alamouti Scheme using PPM.................................... 26

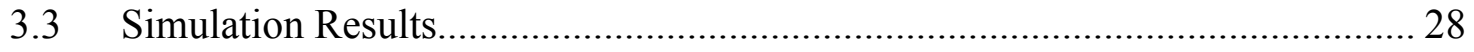

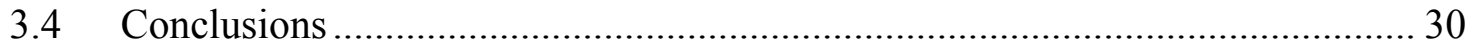

4. FULL DIVERSITY QOSTBC FOR PPM SIGNALS ............................................. 32

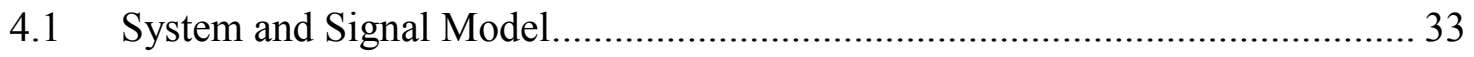

4.2 STBC from Quasi-Orthogonal Design.................................................... 35

4.3 Constellation Rotation for Full Diversity QOSTBC Systems.......................... 36

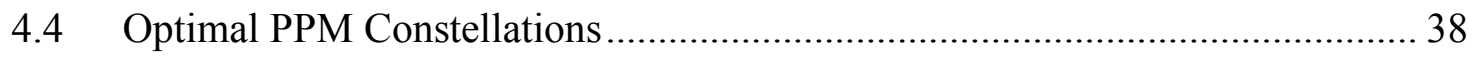

4.5 Simulation Results and Discussion .............................................................. 44

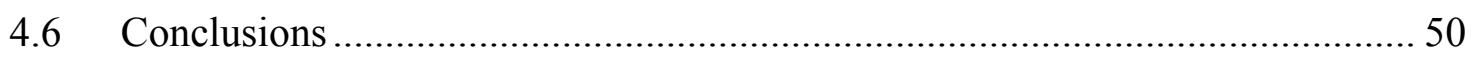

5. SYMBOL-WISE DECODING FOR QOSTBC ENCODED PPM SIGNALS ........... 51

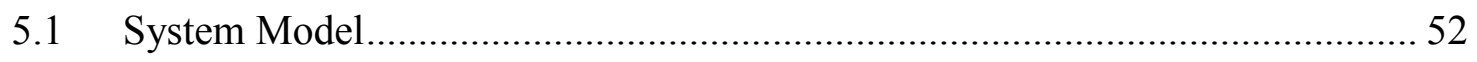

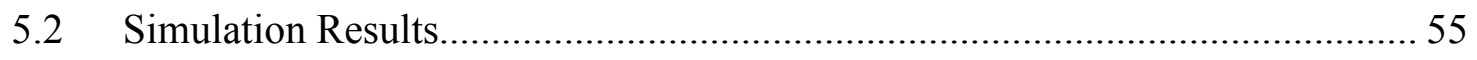

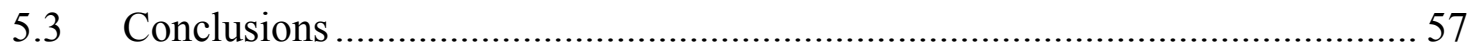


6. CONCLUSIONS AND FUTURE WORK

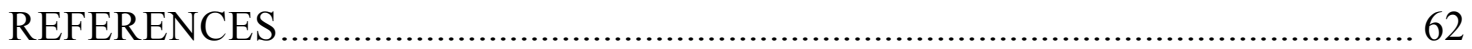

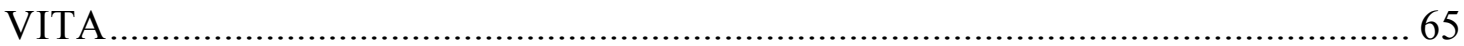




\section{LIST OF TABLES}

TABLE

PAGE

2.1 Euclidean Distance between Symbols for Linear-Binary PPM ................................ 9

2.2 Polynomial Phase Coefficient Mapping for Linear-Binary PPM ............................. 9

2.3 Polynomial Phase Coefficient Mapping for $2^{\text {nd }}$ Order-Binary PPM …..................... 10

2.4 Polynomial Phase Coefficient Mapping for $3^{\text {rd }}$ Order-Binary PPM......................... 11

4.1Comparison of $\mathrm{CGD}_{\min }$ and $C I C_{\max }$ values for PPM and PSK Constellations............ 43

4.2 BER Performance for QOSTBC using $3^{\text {rd }}$ Order Binary-PPM for Different Rotation Angles 


\section{LIST OF FIGURES}

FIGURE

PAGE

2.1 Optimum Maximum Likelihood Receiver for AWGN Channels 12

2.2 BER Performance of Linear-Binary PPM with and without Gray Bit Labeling 17

2.3 BER Performance of $2^{\text {nd }}$ Order-Binary PPM with and without Gray Bit Labeling... 18

2.4 BER Performance of 3rd Order-Binary PPM with and without Gray Bit Labeling... 18

2.5 BER Performance Comparison for Linear-Binary PPM using High-Order Ambiguity Function (HAF), Suboptimal Maximum Likelihood (S-ML), and Maximum Likelihood (ML) Demodulators 19

2.6 BER Performance Comparison for 2nd Order-Binary PPM using High-Order Ambiguity Function (HAF), Suboptimal Maximum Likelihood (S-ML), and Maximum Likelihood (ML) Demodulators

2.7 BER Comparison between Linear-Binary PPM and QPSK .................................. 20

2.8 BER Comparison between $2^{\text {nd }}$ Order-Binary PPM and 8PSK ................................ 20

2.9 BER Comparison between $3^{\text {rd }}$ Order-Binary PPM and 16PSK .............................. 21

3.1. Transmitter and Receiver Model for PPM-OSTBC ................................................ 27

3.2 BER Performance Comparison of SISO Linear-binary PPM, Linear-Binary PPMOSTBC and QPSK-OSTBC ........................................................................ 29

3.3 BER Performance Comparison of $2^{\text {nd }}$ Order-Binary PPM-OSTBC and 8PSKOSTBC 30

3.4 BER Performance Comparison of $3^{\text {rd }}$ Order-Binary PPM-OSTBC and 16PSKOSTBC

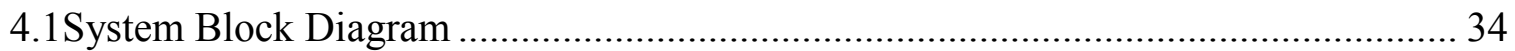

4.2 CGD min $(\phi(t))$ for Rotations on the Constant and First-Order Coefficients ............ 39

4.3 CGDmin and CICmax vs. Rotation Angle for Linear-binary PPM ......................... 41

4.4 CGD min and CICmaxvs. Rotation Angle for $2^{\text {nd }}$ Order-Binary PPM ..................... 42 
4.5 CGDminand CICmaxvs. Rotation Angle for $3^{\text {nd }}$ Order-Binary PPM.

4.6 BER Performance of Linear-Binary PPM with QOSTBC for Different Rotation Angles

4.7 BER Performance for QOSTBC using Linear- Binary PPM and QPSK 45

4.8 BER Performance for QOSTBC using $2^{\text {nd }}$ Order-Binary PPM for Different Rotation Angles

4.9 BER Performance for QOSTBC using Full Diversity 8PSK and $2^{\text {nd }}$ Order-Binary PPM

4.10 BER Performance for QOSTBC using $3^{\text {rd }}$ Order-Binary PPM for Different Rotation Angles

4.11 BER Performance for QOSTBC using Full Diversity 16PSK and $3^{\text {rd }}$ Order-

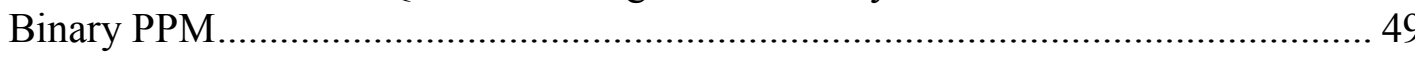

5.1. Transmitter and Receiver Model for PPM-QOSTBC using Quasi-ZF...................... 52

5.2. BER Performance Comparison of Linear-Binary PPM-QOSTBC and QPSK-

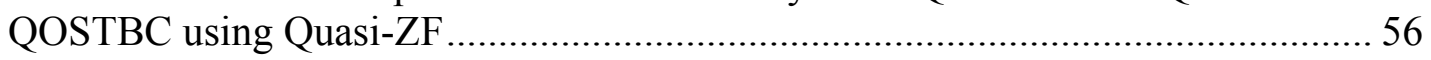

5.3. BER Performance Comparison of Quadratic-Binary PPM-QOSTBC and 8PSK-

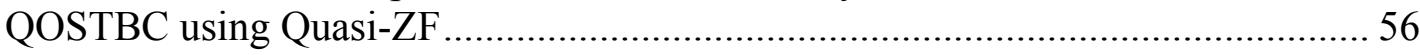

5.4. BER Performance Comparison of $3^{\text {rd }}$ Order-Binary PPM-QOSTBC and 16PSKQOSTBC using Quasi-ZF. 


\section{LIST OF ABBREVIATIONS}

TERM / UNIT OF MEASUREMENT

Multiple-Input Multiple Output

Space-Time Block Coding

Orthogonal Space-Time Block Coding

Quasi-Orthogonal Space-Time Block Coding

Polynomial Phase Modulation

Signal-to-Noise Ratio

Bit Error Rate

interference cancellation

Coding Gain Distance

Channel Interference Coefficient

High-Order Ambiguity Function

Product High-Order Ambiguity Function

Maximum-Likelihood

Additive white Gaussian noise

channel side information

minimum mean square error

Maximal Ratio Combining

log-likelihood ratio

software defined radio

Phase Shift Keying

Power Spectral Density
SYMBOL / ABBREVIATION

MIMO

STBC

OSTBC

QOSTBC

PPM

SNR

BER

IC

CGD

CIC

HAF

PHAF

ML

AWGN

CSI

MMSE

MRC

LLR

SDR

PSK

PSD 
Mobile Terminal $\quad$ MT

Quadrature Amplitude Modulation $\quad$ QAM

Single-Input Single-Output $\quad$ SISO

Binary Phase Shift Keying $\quad$ BPSK

Differential Binary Phase Shift Keying DBPSK

Line Of Sight $\quad$ LOS

Radio Frequency RF

Long Term Evolution $\quad$ LTE 


\section{CHAPTER 1}

\section{INTRODUCTION}

Current commercial communication systems are required to support a wide range of applications. As the number of services that wireless mobile devices (such as cell phones, BlackBerries, and other types of smart phones) provide increases, the need to make available higher bandwidth and battery life for such devices becomes more critical. Traditionally, systems with one antenna at the transmitter and one at the receiver, also known as single-input single-output (SISO) systems, have been used for most wireless applications; however, such systems have been shown to be less energy efficient than systems using more than one antenna at both ends of the communication link [1]. The reason for this lack of energy efficiency is that SISO systems are particularly vulnerable to the deep fading characteristics of the mobile wireless channel and therefore require more average energy per symbol to ensure a specific error rate. As transmission rates increase the total energy needed also increases and for SISO systems this ultimately leads to a steep reduction of the wireless device's battery life. To address some of these issues, multiple antenna communication systems have recently gained significant attention due to the increased transmission rates and reliability they provide with respect to their single antenna counterparts. In fact, many modern communication systems such as Third Generation (3G) cellular systems, wireless local area networks (WLAN), wireless metropolitan area networks (WMAN), long term evolution (LTE) advanced, and worldwide interoperability for microwave access (WiMAX) use multiple antenna techniques to exploit their benefits in order to take advantage of the increased capacity that these provide. 
Orthogonal space-time block coding (STBC) is one of such multiple antenna techniques that have drawn attention from researchers in recent years. Due to the diversity gain they provide to communication systems, these codes are used to more effectively exploit the multi-path characteristic of the wireless channel [2]. For two transmit antennas, Alamouti in [3] provided a simple scheme in which an orthogonal design is employed to provide both full diversity and simple single symbol maximum likelihood decoding. It has been shown, however, that orthogonal codes for complex constellations and more than two transmit antennas cannot achieve full transmission rate [4]. For this reason, Jafarkhani in [4] proposed a code structure in which the columns of the transmission matrix are divided into groups. While the columns within a group are not orthogonal to each other, different groups are orthogonal to each other. This code structure is known as quasi-orthogonal STBCs (QOSTBCs) and although such codes guarantee full transmission rate, they do not provide full diversity or simple symbol-wise ML decoding.

In [5], Su and Xia presented quasi-orthogonal STBCs that achieve full rate and full diversity. There, it was shown that such characteristics can be achieved by optimally selecting a constellation rotation angle based on the maximization of the diversity product. Specifically, half of the symbols transmitted in one signaling interval are chosen from the rotated constellation, while the other half are chosen from the unmodified constellation. In [5], [6], and [7] the optimal rotation angles for PSK and QAM constellations are presented. There it was demonstrated that the QOSTBCs employing constellation rotation have an improved bit-error rate (BER) performance over the QOSTBC systems without it. However, using PSK or QAM does not guarantee the best possible error rate performance in highly mobile scenarios. 
To improve BER performance of single antenna systems and thus increase the battery life of such systems, Sinha et al. in [8] introduced a power efficient modulation format in which the coefficients of polynomial phase functions were used to carry information. This modulation format, known as polynomial phase modulation (PPM), when compared to PSK and QAM, was shown to provide significant improvements in BER performance under AWGN channels.

Following the results in [8] for single input single output (SISO) systems, Dam et al. in [9] proposed a space-time module structure for PPM signals. The proposed module structure allows for the simple design of real orthogonal space-time codes for more than two transmit antennas because the encoding is performed on the phase coefficients. Nevertheless, in [9], it is also shown that the error rate performance of such structure is worse than conventional modulation formats at high signal-to-noise ratios (SNR). The reason behind this decline in performance is that by encoding only the phase coefficients, the module structure does not ensure that the transmitted signals will provide full transmit diversity.

\subsection{Objective and Contributions}

Polynomial phase modulated signals are a promising alternative to conventional modulation formats such as M-ary PSK because of their lower error rates in additive white Gaussian noise (AWGN) and Rayleigh fading channels. Sinha et al. presented this modulation technique for a single-input single-output (SISO) system and evaluated its performance under different channel conditions when the high order ambiguity function (HAF), also known as the polynomial phase transform (PPT) [10] [11], was used as a 
decoding method [8]. However, in the error rate performance study shown in [8], the carrier power is used for BER calculations instead of the average energy per bit, which leads a more accurate indication of the system's power efficiency.

In this dissertation three different decoding methods for SISO PPM systems are studied. Specifically, a maximum likelihood (ML), a sub-optimal maximum likelihood (S-ML) [16] [19], and a HAF-based decoder, such as the one in [8], are considered. These decoding structures are based on common techniques used for parameter estimation of polynomial phase signals for radar applications [19]; however, here we employ them in the context of digital communication systems. Using Monte Carlo simulations, the evaluation of the error rate performance of each one of these systems is presented and compared to that of M-ary PSK. Assuming normalized average energy per symbol, it is shown that PPM outperforms PSK when an optimal ML decoder is employed. In addition to this, a bit labeling strategy based on gray coding for linear, $2^{\text {nd }}$ order, and $3^{\text {rd }}$ order PPM signals is also presented.

Since MIMO systems have the potential of providing higher transmission rates (multiplexing gain) or improved error rate performances (diversity gain) [2], Sinha extended his results to systems with two transmit antennas. In chapter 3, the bit error rate (BER) performance of such PPM based systems is studied through Monte Carlo simulations. Because the orthogonal space-time block code (OSTBC) in [3] is used as the transmission scheme, symbol-wise decoding is possible, and thus any of the three PPM demodulators from chapter 2 can be used depending on the complexity requirements of 
the system. Again, a comparison of the performance of each one of these decoding methods when applied to PPM OSTBC systems is presented.

As the number of transmit antennas is increased, a higher diversity order is achieved and thus, lower error rates at high signal-to-noise ratios (SNR) can be obtained [18]. Dam in [9] proposed a so called space-time module structure in order to exploit the advantages of using multiple transmit antennas along with PPM signals. This technique allows for simple code design; however, it was shown in [9] that at high SNRs, the BER is of this PPM based system is higher than that of space-time codes using PSK formats. This is probably evidence that the module structure does not ensure the highest diversity order given the number of transmit antennas. In chapter 4, full diversity PPM space-time coded systems for four transmit antennas are presented. Here, we make use of the quasiorthogonal space-time code (QOSTBC) proposed by Jafarkhani in [4]. However, it is well known that such codes do not achieve full diversity unless half of the transmitted symbols are chosen from an optimally rotated constellation. Su et al. in [5] obtained optimal rotation angles for different PSK, QAM, and TRI constellations by maximizing the system's minimum coding gain distance (CGD); nevertheless, to the author's knowledge, no prior work has been done to find such rotation angles for PPM signals. Also, since the maximization of the minimum CGD does not yield a unique optimal rotation angle, in this work a new metric known a channel interference coefficient (CIC) is proposed. The CIC is a factor that is multiplied by the channel matrix components during optimal ML decoding. It, therefore, has a direct effect on the ultimate error rate performance of the system. That is, ensuring that the value of this parameter is as low as possible guarantees that the interference caused by the channel terms is also low and thus, 
an improved error rate performance can be obtained. In this dissertation it is shown through Monte Carlo simulations that selecting the angle that yields the best trade-off between the minimum CGD and the maximum CIC ensures full diversity and allows for a better error rate performance than when only the minimum CGD is considered.

One of the main disadvantages of QOSTBCs is that decoding of the transmitted information can only be performed through a pair-wise ML algorithm [20]. This is inconvenient for PPM signals as lower complexity decoding techniques such as S-ML or HAF cannot be employed. In chapter 5, the quasi zero-forcing (ZF) technique introduced by Jeong in [15] is applied to the Jafakhani QOSTBC in order to decouple the received symbols and enable symbol-wise decoding. At that point, the lower complexity PPM decoding algorithms are applied and their BER performance is evaluated and compared to that of PSK systems using the same quasi-ZF method. 


\section{CHAPTER 2}

\section{POLYNOMIAL PHASE MODULATION}

In this chapter, a review of polynomial phase modulated signals is presented. The mathematical model of such signals is illustrated and different demodulation methods based on maximum-likelihood (ML) and the high-order ambiguity function (HAF) are explained. A comparison of the performance of each of these methods is provided through Monte-Carlo simulations at the end of the chapter.

\subsection{Signal Model}

A polynomial phase modulated signal $s(t)$ for a symbol period $T_{0}$ is described in [8] as:

$$
s(t)=\sqrt{\frac{2 E_{s}}{T_{0}}} \cos \left(\omega_{c} t+\varphi(t)\right)
$$

where $E_{s}$ is assumed to be the normalized energy per symbol and

$$
\varphi(t)=a_{M}\left(t / T_{0}\right)^{M}+a_{M-1}\left(t / T_{0}\right)^{M-1}+\cdots+a_{1}\left(t / T_{0}\right)+a_{0}
$$

for $0 \leq t \leq T_{0}$ is the time-varying phase whose phase coefficients are chosen from $a_{m}$ $\in\{ \pm \pi / 2\}$. Therefore, depending on the transmitted symbol, a specific combination of phase coefficients is selected. From Equation (2.1), it can be seen that one could increase the transmission rate of a PPM system by increasing the order of the polynomial phase function in order to accommodate more bits per symbol. It is also important to note that although the value of the polynomial phase coefficients can be chosen arbitrarily, it has been shown in [21] that selecting these values from the set $\{ \pm \pi / 2\}$ yields PPM signals without discrete components in their spectrum and with similar spectral characteristics as PSK signals with the same transmission rate. This ensures fairness in the error rate 
performance comparisons that will follow later on in this chapter and throughout this dissertation.

In addition, throughout this work, the complex analytical form will be used to describe polynomial phase modulated signals. Based on the Hilbert transform, a model of the PPM signal in Equation (2.1) can also be written as [8]:

$$
s(t)=\sqrt{\frac{2 E_{s}}{T_{0}}} \exp \left(i\left(\omega_{c} t+\varphi(t)\right)\right)
$$

\subsection{Gray Bit Labeling for Polynomial Phase Modulated Signals}

Gray bit labeling has been extensively studied for specific constellations of modulation formats such as PSK and QAM with the aim of reducing the number of bit errors incurred on at the receiver end [22]. Based on the minimum Euclidean distance between pairs of signal points, in this section a Gray bit labeling approach for PPM signals of different orders is presented. As previously mentioned, this technique is used to reduce the number of bit errors by ensuring adjacent symbols differ by only one bit.

Assuming the signals $s_{m}(t)$ and $s_{n}(t)$, for $1 \leq m \leq M$ and $1 \leq n \leq M$, belong to an Mary PPM signal set, then the squared Euclidean distance between all possible pairs of symbols is calculated using the following definition for $m \neq n$ :

$$
d^{2}=\int_{0}^{T_{0}}\left(s_{m}(t)-s_{n}(t)\right)^{2} d t
$$

The Euclidean distance for each symbol of a linear-binary PPM signal set shown in Table 2.1 was found using the above formulation. Notice that one could arrive to the same results by obtaining the signal space representation of the linear-binary PPM constellation. 
After that, Gray bit labeling was performed so that the closest symbols would differ by only one bit. The resulting bit labeling strategy is shown in Table 2.1 .

In the table, it is observed that since initially symbols represented by $\left\{a_{0}=-\pi / 2\right.$, $\left.a_{1}=-\pi / 2\right\}$ and $\left\{a_{0}=\pi / 2, a_{1}=\pi / 2\right\}$ are adjacent to each other and the number of bit changes from one to the other was two, a viable approach is to change the symbol labeling from 11 to 10 and from 10 to 11 . Notice that this technique effectively reduces the number of bit changes from adjacent PPM symbols to one.

Table 2.1 Euclidean Distance between Symbols for Linear-Binary PPM

\begin{tabular}{|c|c|c|c|c|}
\hline & $\mathbf{0 0}$ & $\mathbf{0 1}$ & $\mathbf{1 0}$ & $\mathbf{1 1}$ \\
\hline $\mathbf{0 0}$ & - & $\sqrt{2}$ & 2 & $\sqrt{2}$ \\
\hline $\mathbf{0 1}$ & $\sqrt{2}$ & - & $\sqrt{2}$ & 2 \\
\hline $\mathbf{1 0}$ & 2 & $\sqrt{2}$ & - & $\sqrt{2}$ \\
\hline $\mathbf{1 1}$ & $\sqrt{2}$ & 2 & $\sqrt{2}$ & - \\
\hline
\end{tabular}

Table 2.2 Polynomial Phase Coefficient Mapping for Linear-Binary PPM

\begin{tabular}{|c|c|c|c|}
\hline Binary Word & $\boldsymbol{a}_{\mathbf{0}}$ & $\boldsymbol{a}_{\mathbf{1}}$ & Gray Labeling \\
\hline 00 & $-\pi / 2$ & $-\pi / 2$ & 00 \\
\hline 01 & $-\pi / 2$ & $\pi / 2$ & 01 \\
\hline 10 & $\pi / 2$ & $-\pi / 2$ & 11 \\
\hline 11 & $\pi / 2$ & $\pi / 2$ & 10 \\
\hline
\end{tabular}


The same approach used to obtain the Gray labeling representation for the linear-binary PPM set was also applied to second and third order binary PPM signals. The resulting mappings are presented in Table 2.3 and Table 2.4 respectively.

Table 2.3 Polynomial Phase Coefficient Mapping for $2^{\text {nd }}$ Order-Binary PPM

\begin{tabular}{|c|c|c|c|c|}
\hline Binary Word & $\boldsymbol{a}_{\mathbf{0}}$ & $\boldsymbol{a}_{\mathbf{1}}$ & $\boldsymbol{a}_{\mathbf{2}}$ & Gray Labeling \\
\hline 000 & $-\pi / 2$ & $-\pi / 2$ & $-\pi / 2$ & 000 \\
\hline 001 & $-\pi / 2$ & $-\pi / 2$ & $\pi / 2$ & 001 \\
\hline 010 & $-\pi / 2$ & $\pi / 2$ & $-\pi / 2$ & 011 \\
\hline 011 & $-\pi / 2$ & $\pi / 2$ & $\pi / 2$ & 010 \\
\hline 100 & $\pi / 2$ & $-\pi / 2$ & $-\pi / 2$ & 100 \\
\hline 101 & $\pi / 2$ & $-\pi / 2$ & $\pi / 2$ & 101 \\
\hline 110 & $\pi / 2$ & $\pi / 2$ & $-\pi / 2$ & 111 \\
\hline 111 & $\pi / 2$ & $\pi / 2$ & $\pi / 2$ & 110 \\
\hline
\end{tabular}

\subsection{Optimum PPM Demodulator using Maximum-Likelihood Estimation}

Assuming the PPM signal, $s(t)$, is transmitted through an additive white Gaussian noise (AWGN) channel, the received signal will have the form:

$$
r(t)=s(t)+\eta(t)
$$

where $\eta(t)$ represents the thermal noise at the receiver and it is modeled as AWGN.

If a vector of $N$ observations of the received sequence is denoted by $\boldsymbol{r}$, and given that all the possible transmitted sequences, $\boldsymbol{s}_{\boldsymbol{m}}$, have the same probability, then the transmitted PPM signal can be optimally estimated by finding the sequence for which the conditional probability, $P\left(\boldsymbol{s}_{\boldsymbol{m}} \mid \boldsymbol{r}\right)$, is maximized [18]. That is, 


$$
\hat{\boldsymbol{s}}_{\boldsymbol{i}}=\arg \max _{\boldsymbol{s}_{i}} P\left(\boldsymbol{s}_{\boldsymbol{m}} \mid \boldsymbol{r}\right)
$$

where $m=1, \ldots, M$.

In [18] it is shown that this decision is equivalent to finding the signal $\boldsymbol{s}_{\boldsymbol{m}}$ that minimizes the Euclidean distance metric:

$$
D\left(\boldsymbol{r}, \boldsymbol{s}_{\boldsymbol{m}}\right)=\sum_{k=1}^{N}\left(r_{k}-s_{m k}\right)^{2}
$$

where $r_{k}$ represents the $k$ th observation of a received sequence of length $N$.

Table 2.4 Polynomial Phase Coefficient Mapping for $3^{\text {rd }}$ Order-Binary PPM

\begin{tabular}{|c|c|c|c|c|c|}
\hline Binary Word & $\boldsymbol{a}_{\mathbf{0}}$ & $\boldsymbol{a}_{\mathbf{1}}$ & $\boldsymbol{a}_{\mathbf{2}}$ & $\boldsymbol{a}_{\mathbf{3}}$ & Gray Labeling \\
\hline 0000 & $-\pi / 2$ & $-\pi / 2$ & $-\pi / 2$ & $-\pi / 2$ & 0000 \\
\hline 0001 & $-\pi / 2$ & $-\pi / 2$ & $-\pi / 2$ & $\pi / 2$ & 0001 \\
\hline 0010 & $-\pi / 2$ & $-\pi / 2$ & $\pi / 2$ & $-\pi / 2$ & 0100 \\
\hline 0011 & $-\pi / 2$ & $-\pi / 2$ & $\pi / 2$ & $\pi / 2$ & 0010 \\
\hline 0100 & $-\pi / 2$ & $\pi / 2$ & $-\pi / 2$ & $-\pi / 2$ & 0011 \\
\hline 0101 & $-\pi / 2$ & $\pi / 2$ & $-\pi / 2$ & $\pi / 2$ & 0101 \\
\hline 0110 & $-\pi / 2$ & $\pi / 2$ & $\pi / 2$ & $-\pi / 2$ & 0111 \\
\hline 0111 & $-\pi / 2$ & $\pi / 2$ & $\pi / 2$ & $\pi / 2$ & 0110 \\
\hline 1000 & $\pi / 2$ & $-\pi / 2$ & $-\pi / 2$ & $-\pi / 2$ & 1000 \\
\hline 1001 & $\pi / 2$ & $-\pi / 2$ & $-\pi / 2$ & $\pi / 2$ & 1001 \\
\hline 1010 & $\pi / 2$ & $-\pi / 2$ & $\pi / 2$ & $-\pi / 2$ & 1100 \\
\hline 1011 & $\pi / 2$ & $-\pi / 2$ & $\pi / 2$ & $\pi / 2$ & 1010 \\
\hline 1100 & $\pi / 2$ & $\pi / 2$ & $-\pi / 2$ & $-\pi / 2$ & 1011 \\
\hline 1101 & $\pi / 2$ & $\pi / 2$ & $-\pi / 2$ & $\pi / 2$ & 1101 \\
\hline 1110 & $\pi / 2$ & $\pi / 2$ & $\pi / 2$ & $-\pi / 2$ & 1111 \\
\hline 1111 & $\pi / 2$ & $\pi / 2$ & $\pi / 2$ & $\pi / 2$ & 1110 \\
\hline & & & & & \\
\hline
\end{tabular}


In addition to this, one can also show that, assuming the energy of all possible transmitted signals is equal, the maximum likelihood (ML) decoder is equivalent to maximizing the correlation metrics

$$
C\left(\boldsymbol{r}, \boldsymbol{s}_{\boldsymbol{m}}\right)=\int_{0}^{T_{0}} r(t) s_{m}(t) d t .
$$

The diagram for this type of decoder is illustrated in Fig. 0.1. It is important to note that, as shown in equation (2.6), the correct estimation of a transmitted sequence depends on the Euclidean distance of that symbol to all other possible symbols within a modulation format. Therefore, the error rate of this demodulation scheme also depends on such distance.

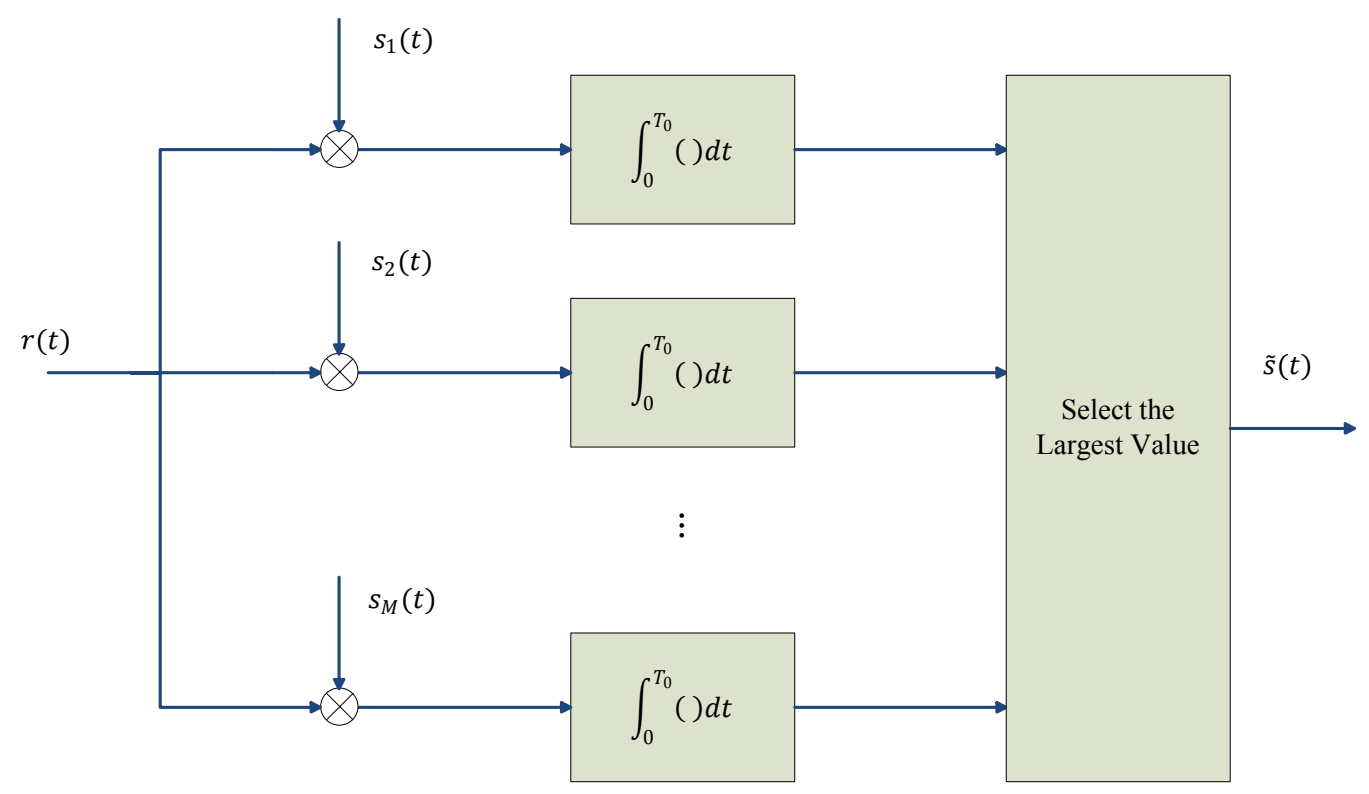

Fig. 0.1 Optimum Maximum Likelihood Receiver for AWGN Channels

\subsection{Suboptimal Maximum-Likelihood Demodulation}

The demodulation of PPM signals can also be performed through the estimation of the polynomial phase coefficients $\left\{a_{M}, a_{M-1}, \ldots, a_{1}, a_{0}\right\}$.The following algorithm, introduced by Boashash in [16], performs such estimation by first using a maximum-likelihood (ML) 
decision rule for the high-order coefficients. It then uses the estimated values to unwrap the constant phase coefficient and finds its value by calculating the angle of the unwrapped function. Since the grid search dimension for an Mth order PPM signal is now reduced from $M+1$ to $M$, this method allows for a lower complexity demodulation process, compared to the optimum ML algorithm presented in the previous section, at the expense of poorer error rate performance. This technique is illustrated as follows:

After down conversion and sampling, the received finite length polynomial phase sequence can be written as:

$$
r(n)=\operatorname{Aexp}(j \varphi(n))+\eta(n)
$$

where $\eta(n)$ is additive white Gaussian noise with zero mean. The phase polynomial is given as $\varphi(n)=a_{M}(n \Delta)^{M}+a_{M-1}(n \Delta)^{M-1}+\cdots+a_{1}(n \Delta)+a_{0}$, for $\quad 0 \leq n \leq N-1$ and $N$ samples. $\Delta$ is the sampling interval and is obtained from $\Delta=T_{0} / N$.

Then, the phase coefficients of the PPM signal can be found using the ML estimator given by

$$
\left(\hat{a}_{M}, \hat{a}_{M-1}, \ldots, \hat{a}_{1}\right)=\arg \max _{a_{M}, a_{M-1}, \ldots, a_{1}}\left|\sum_{n=0}^{N-1} r(n) \exp \left(-j \sum_{m=1}^{M} a_{m}(n \Delta)^{m}\right)\right| .
$$

The constant phase term can be found from

$$
\hat{a}_{0}=\operatorname{angle}\left\{\sum_{n=0}^{N-1} r(n) \exp \left(-j \sum_{m=1}^{M} \hat{a}_{m}(n \Delta)^{m}\right)\right\}
$$

Since the alphabet is finite $\left(a_{m} \in\{ \pm \pi / 2\}\right)$, the complexity of the estimator given in (2.9) is small for low order polynomials; however, just as in the case of the optimal ML decoder, as the order increases, the algorithm complexity grows exponentially due to the multidimensional grid search required for decoding. 


\subsection{Parameter Estimation Using the High-Order Ambiguity Function}

Because of the complexity of the ML algorithm, we also consider a suboptimal estimation method called polynomial phase transform, later known as the high-order ambiguity function (HAF) [10]. In this method, the polynomial phase coefficients are estimated sequentially. In other words, the highest order coefficient is estimated first. Given the received signal in (2.8), the HAF algorithm is defined recursively as:

$$
\begin{gathered}
r_{1}(n ; \tau)=r(n) \\
r_{2}(n ; \tau)=r_{1}(n ; \tau) r_{1}^{*}(n-\tau) \\
\vdots \\
r_{M}(n ; \tau)=r_{M-1}(n ; \tau) r_{M-1}^{*}(n-\tau ; \tau)
\end{gathered}
$$

where $\tau$ is a predetermined lag whose optimal value is $\tau=N / M$. Then, the high-order ambiguity function HAF can be calculated from

$$
R_{M}(f, \tau)=\sum_{n=0}^{N-1} r_{M}(n ; \tau) \exp (-i 2 \pi f n \Delta)
$$

The Mth-order phase coefficient is then obtained by finding the frequency $f_{\text {max }}$ of the HAF's highest peak and calculating $a_{M}=\frac{2 \pi f_{\max }}{M !(\tau \Delta)^{M-1}}$. Here, the peak search can be restricted to the frequency values corresponding to the alphabet $a_{m} \in\{ \pm \pi / 2\}$. The estimated Mth-order phase coefficient is then removed using $r_{M-1}=r_{M} \exp \left(-i a_{M}(n \Delta)^{M}\right)$ and the process from (2.11) and (2.12) is repeated until $M$ $=0$. At that point, an estimate of the coefficient $a_{0}$ can be found by using the same method as in the S-ML algorithm in equation (2.10). As it can be seen, the search grid is one-dimensional and as the polynomial order increases, the complexity grows linearly. 
However, because in the HAF-based decoding method the phase coefficients are estimated sequentially and the estimation error propagates from higher to lower order coefficients, it is considered a suboptimal method and thus, there is a penalty to be paid in terms of error rate performance with respect to ML for high-order polynomials, as it will be seen later on.

\subsection{Simulation Results and Discussion}

In this section, the performance of single-input single-output (SISO) PPM systems is evaluated when transmission is performed under a frequency flat Rayleigh fading channel. In Fig. 2.2 through Fig. 2.4, the error rate performance of PPM systems with Gray bit labeling at different transmission rates is compared to equivalent systems employing natural encoding. In Fig. 2.2, a linear-binary PPM system is studied. There, it is observed that bit labeling using Gray encoding leads to a signal-to-noise ratio (SNR) improvement of approximately $1 \mathrm{~dB}$ for a BER of about $10^{-2}$ with respect to the system using natural encoding. The BER performance for $2^{\text {nd }}$ and $3^{\text {rd }}$ order PPM signal sets is also shown in Fig. 2.3 and Fig. 2.4, respectively. As expected, an improvement in the error rate performance can be seen when the proposed bit labeling format is employed. Specifically, for the $2^{\text {nd }}$ order-binary PPM a gain of $1 \mathrm{~dB}$ at an error rate of $10^{-1}$ is achieved, while for the $3^{\text {rd }}$ order-binary PPM the improvement is of about $2 \mathrm{~dB}$ at the same error rate.

Next, the performance of different algorithms used for the demodulation of PPM signals (presented in sections 2.3 to 2.5) is investigated through Monte-Carlo simulations. For these simulations it is assumed that the PPM signals are generated without Gray bit 
labeling and are transmitted over a Rayleigh flat fading channel with additive white Gaussian noise. In Fig. 2.5, the BER curves for different linear-binary PPM systems are displayed. In this figure, it is clear that in order to achieve an error rate of $10^{-1}$ the PPM system using an ML decoder requires $3 \mathrm{~dB}$ less than for the other two schemes, demonstrating that ML is a more power efficient alternative; however, this performance advantage comes at the cost of higher complexity.

Fig. 2.6 shows the performance of a $2^{\text {nd }}$ order-binary PPM system. Just as in the case of the linear-binary PPM system, ML estimation outperforms all other methods. In addition to this, it can be observed that the Suboptimal ML algorithm also provides a more advantageous bit error rate characteristic than the HAF-based approach. Because the polynomial phase coefficient estimation using HAF is performed in a sequential manner, the error in the estimation of the highest order coefficient propagates to the estimation of every lower order coefficient causing an overall increase in BER.

Finally, a comparison between PPM and PSK formats for various modulation levels is presented. For these Monte-Carlo simulations, transmission is again done through a frequency flat Rayleigh fading channel and it is assumed that Gray bit labeling has been used in both, PPM and PSK constellations. In addition to this, the receiver for the PPM system employs the optimal ML decoding algorithm presented in section 2.3.

In Fig. 2.7, the BER curves for linear-binary PPM and QPSK SISO systems are displayed. Because both modulation types exhibit the same Euclidean distances between symbols and since the decoding algorithm used in this simulation depends on such metric, the error rate performance is the same for the two systems. Fig. 2.8 shows a comparison of the error rate performances of $2^{\text {nd }}$ Order PPM and 8PSK. It can be 
observed that the PPM system requires approximately $1 \mathrm{~dB}$ less power than 8PSK in order to achieve an error rate of $10^{-1}$. In this case PPM clearly outperforms 8PSK because of its overall higher Euclidean distances between symbols. This effect can also be observed in Fig. 2.9, where a $3^{\text {rd }}$ Order PPM and a 16PSK system are compared. In this instance a separation of almost $2.5 \mathrm{~dB}$ in the BER curves at an error rate of $10^{-1}$ is discerned. Based on the above evidence, one can conclude that PPM is in general a more power efficient digital modulation scheme than PSK and thus more suitable for power constrained systems.

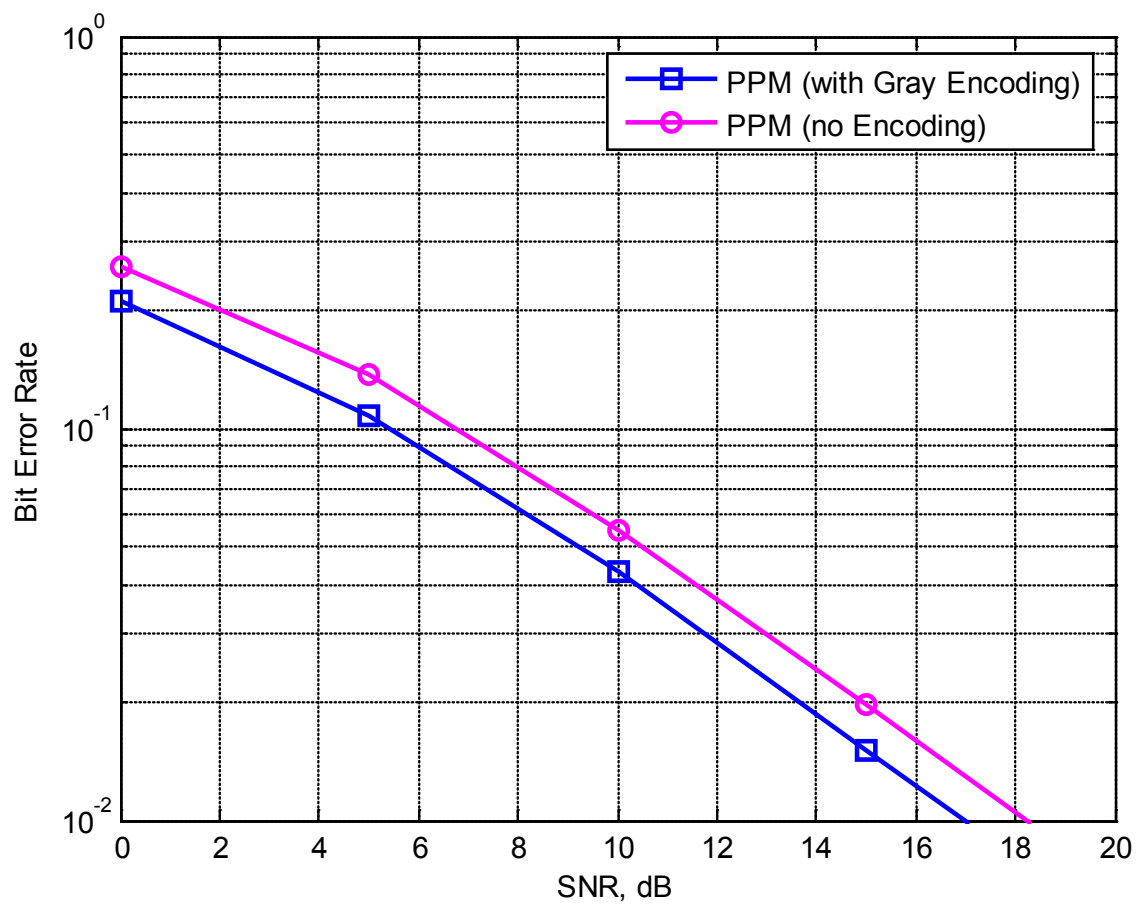

Fig. 2.2 BER Performance of Linear-Binary PPM with and without Gray Bit Labeling 


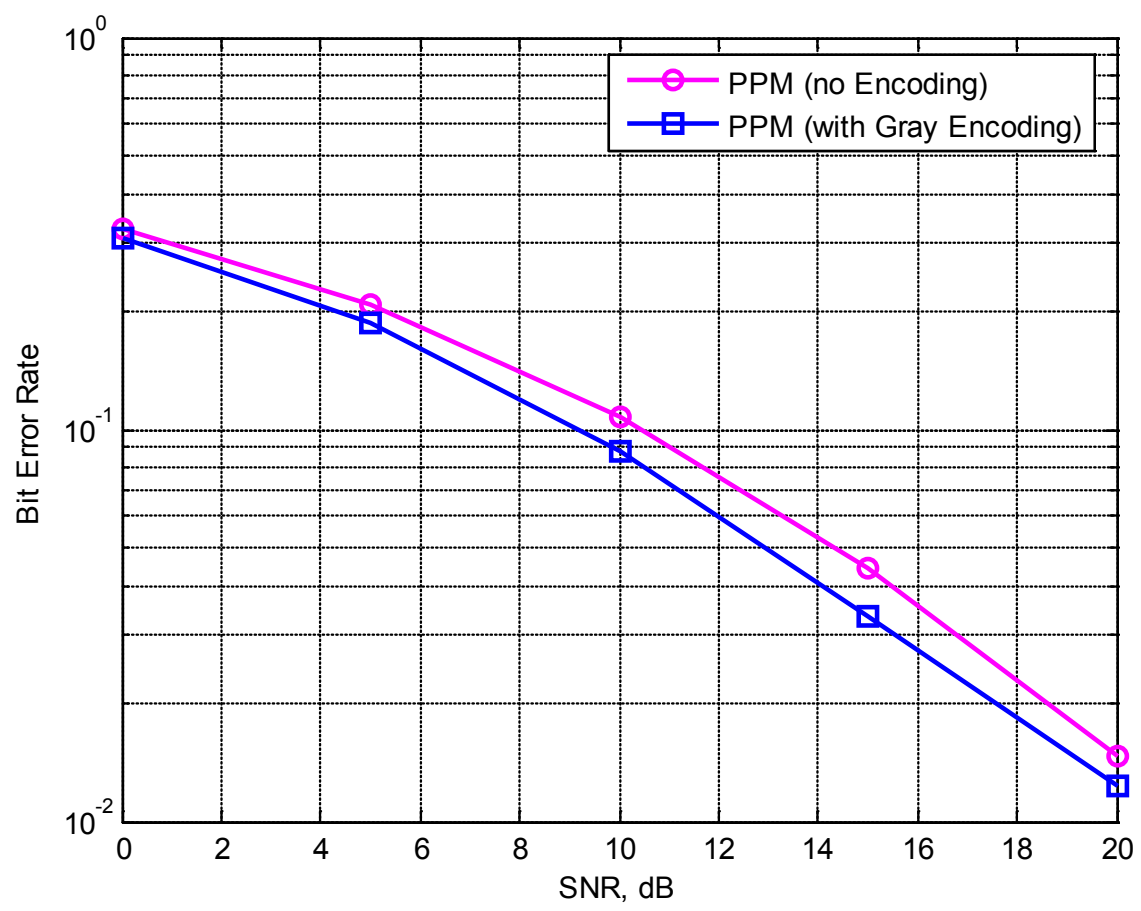

Fig. 2.3 BER Performance of $2^{\text {nd }}$ Order-Binary PPM with and without Gray Bit Labeling

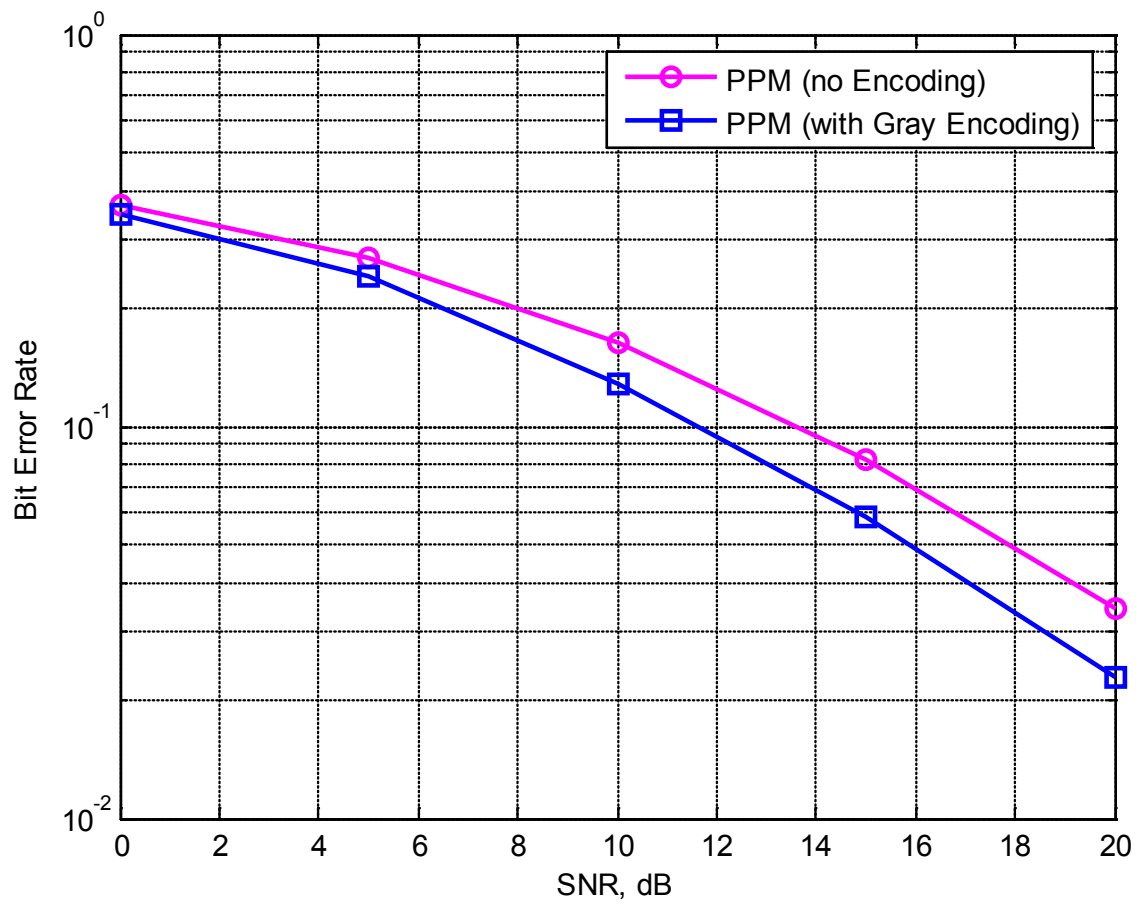

Fig. 2.4 BER Performance of 3rd Order-Binary PPM with and without Gray Bit Labeling 


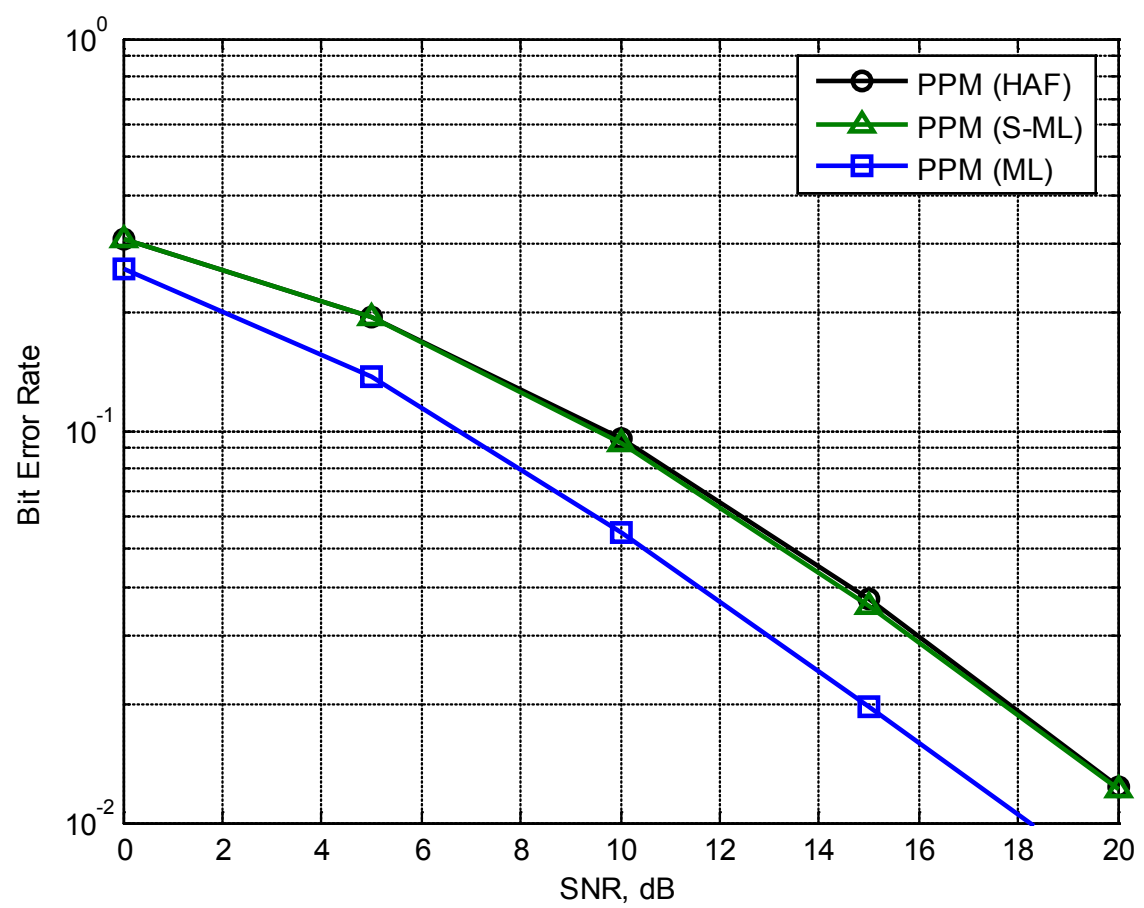

Fig. 2.5 BER Performance Comparison for Linear-Binary PPM using High-Order Ambiguity Function (HAF), Suboptimal Maximum Likelihood (S-ML), and Maximum Likelihood (ML) Demodulators

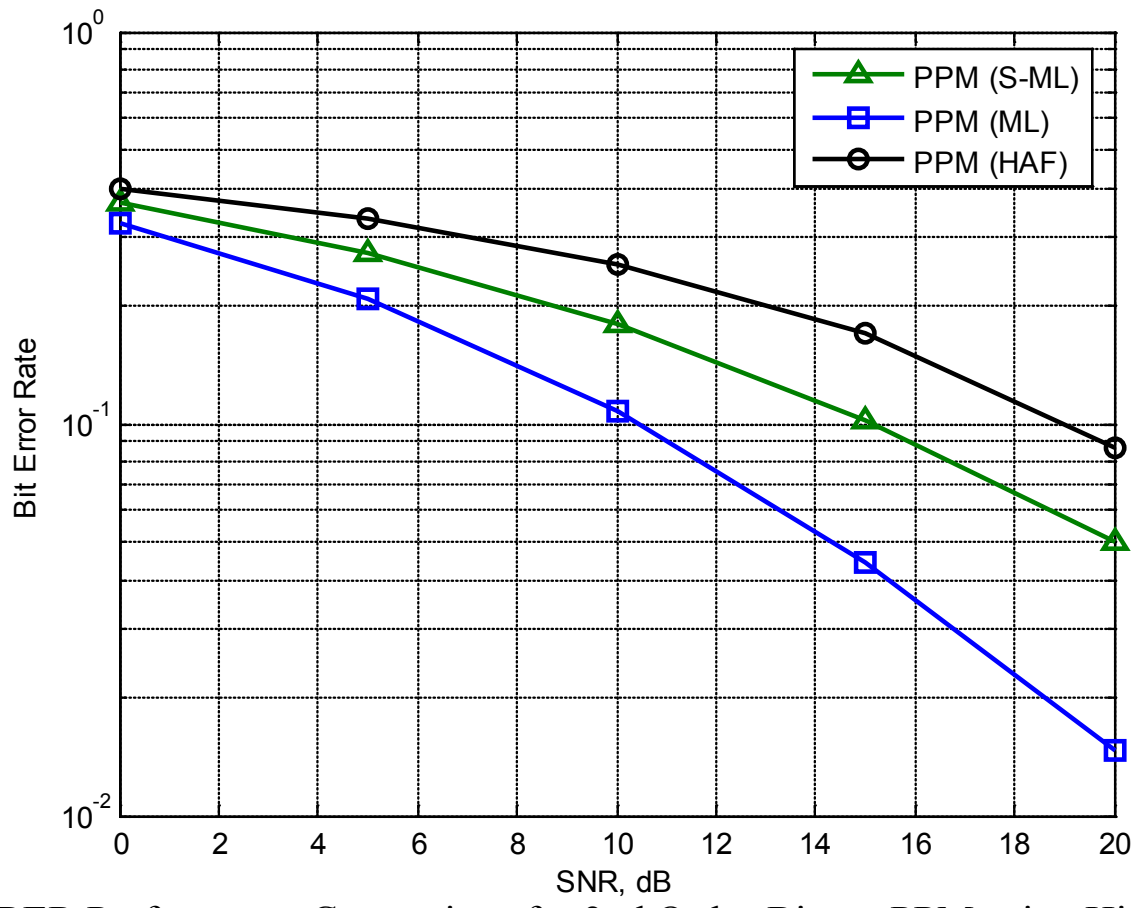

Fig. 2.6 BER Performance Comparison for 2nd Order-Binary PPM using High-Order Ambiguity Function (HAF), Suboptimal Maximum Likelihood (S-ML), and Maximum Likelihood (ML) Demodulators 


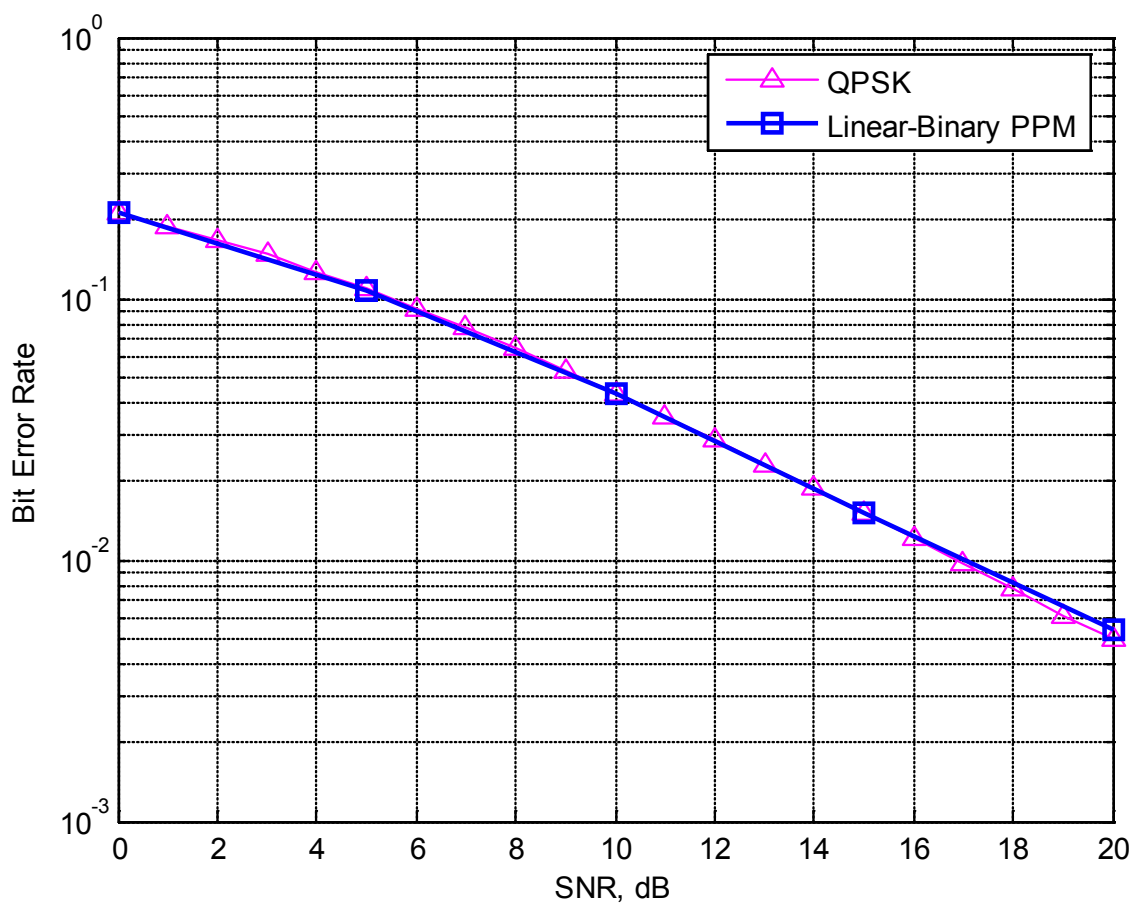

Fig. 2.7 BER Comparison between Linear-Binary PPM and QPSK

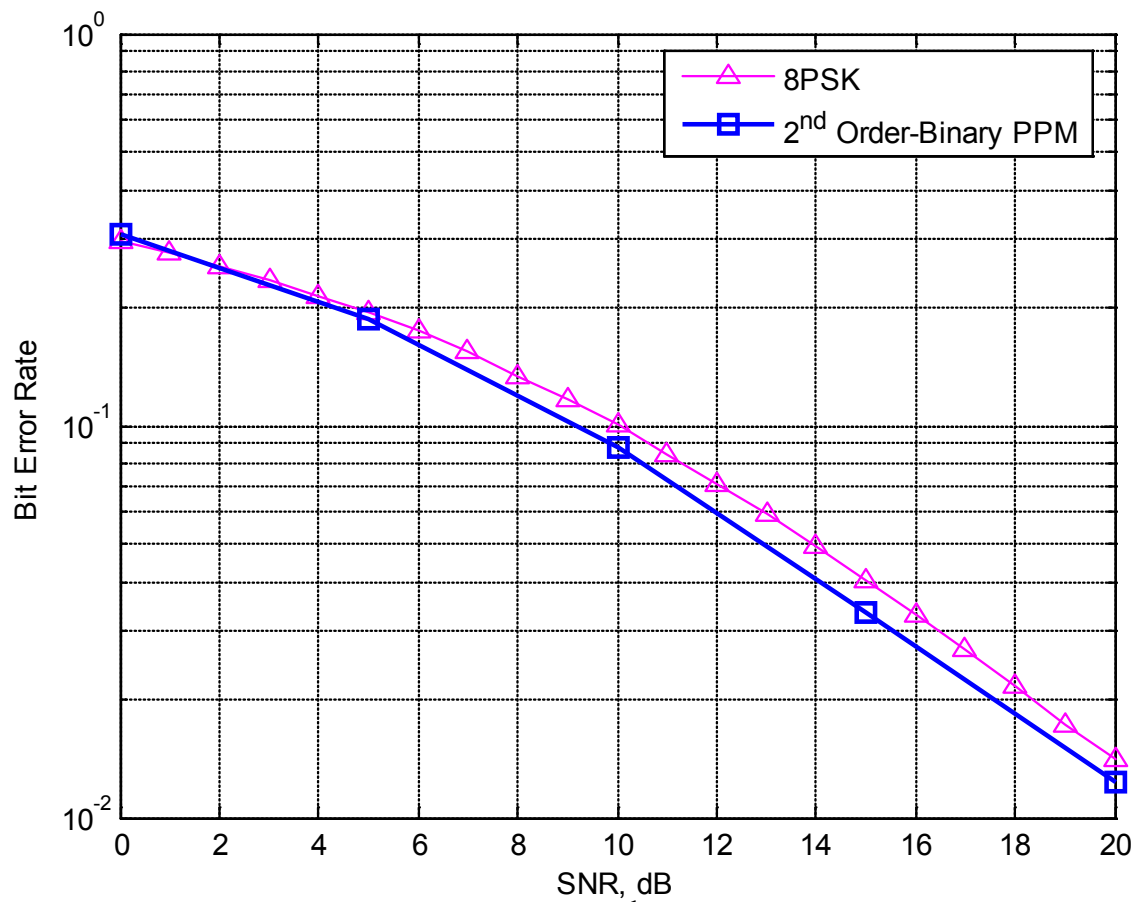

Fig. 2.8 BER Comparison between $2^{\text {nd }}$ Order-Binary PPM and 8PSK 


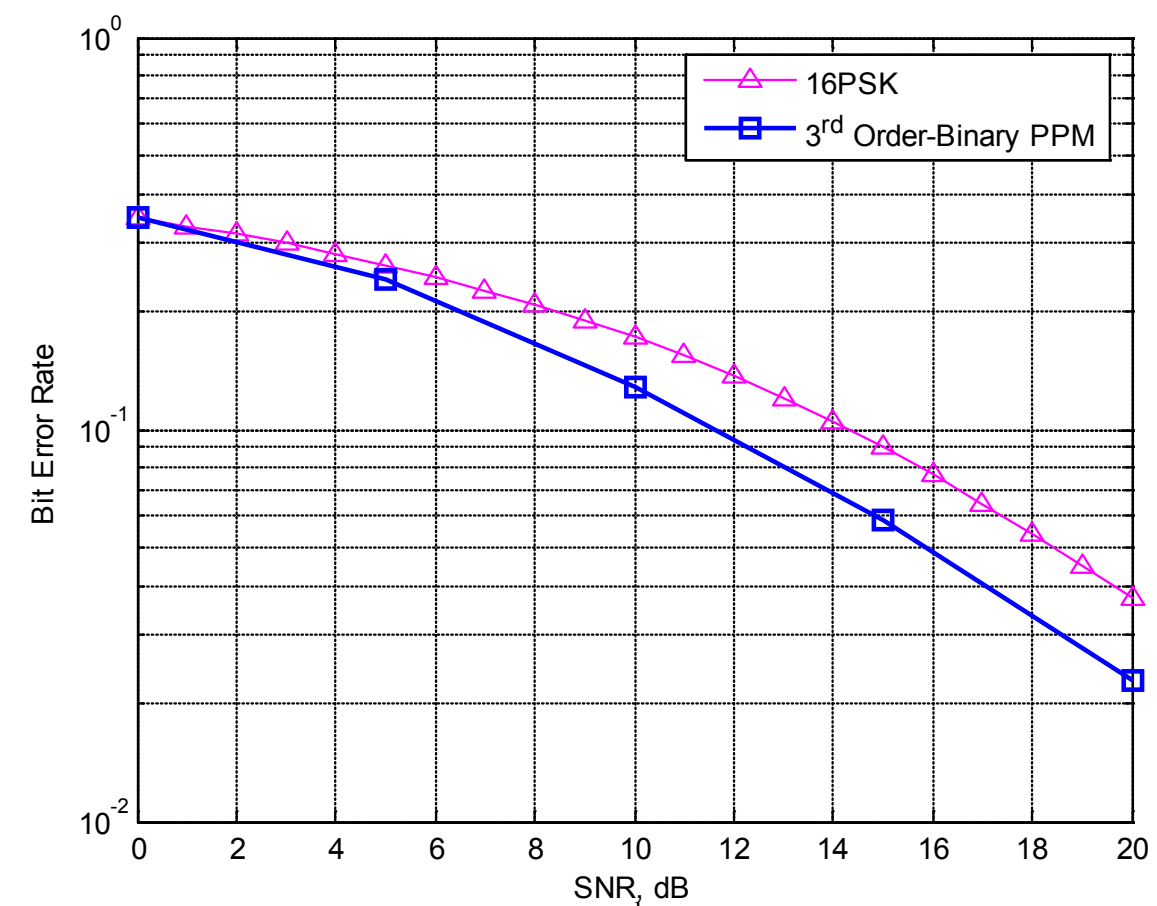

Fig. 2.9 BER Comparison between $3^{\text {rd }}$ Order-Binary PPM and 16PSK

\subsection{Conclusions}

In this chapter, polynomial phase modulation (PPM) was presented as a power efficient alternative to conventional modulation techniques such as phase-shift keying (PSK). Optimal bit labeling using Gray coding was proposed for linear, $2^{\text {nd }}$ order, and $3^{\text {rd }}$ order PPM formats. It was shown that such technique improves the error rate performance of the PPM systems under study when compared to the more intuitive natural bit labeling. The performance of three different decoders, maximum likelihood (ML), Suboptimal maximum likelihood (S-ML), and high-order ambiguity function (HAF), for PPM signals was also investigated. As expected, the ML based decoder showed a much better error rate performance than the other two methods, especially for high order PPM signals. The reason for this is that S-ML and HAF based methods suffer from error propagation from 
the estimation of the high order coefficients. This is particularly prominent in the HAF decoder, where the estimation error propagates from coefficient to coefficient. Nevertheless, it is important to point out that the HAF based receiver requires a less complex system than the other two algorithms because the coefficient search grid is one dimensional regardless of the PPM order.

Lastly, a comparison between PPM and PSK modulation formats was performed. There, PPM signals were shown to be a more power efficient format than PSK mainly at high transmission rates. This highlights the potential of this modulation type in modern wireless communications systems where transmission of information at high speeds under size, weight, and power (SWaP) constraints is the norm. 


\section{CHAPTER 3}

\section{ALAMOUTI ENCODED SYSTEM FOR PPM SIGNALS}

From the discussion and the results presented in the previous chapter, it is clear that polynomial phase modulated (PPM) signals have the potential to provide a lower error rate for a given signal-to-noise ratio than M-ary PSK constellations. This property is advantageous as PPM systems require less power to transmit information at the same rate as PSK systems. Since it has been widely proven that additional improvements in BER performance and capacity can be obtained when transmit diversity techniques for MIMO systems are employed, in this chapter the results from the SISO system presented in the previous chapter are extended to an Alamouti encoded system with two transmit and one receive antennas. The performance of the proposed system is studied and compared to that of an Orthogonal Space Time Block Coding (OSTBC) system using PSK constellations. The argument for justifying the use multiple antennas is presented in the first section. Then, the overall model for the proposed system and simulation results which demonstrate the improved system's BER performance are presented in the following sections.

\subsection{Multiplexing and Diversity in MIMO Systems}

Multiple antenna communication systems have been shown to provide many benefits with respect to their single antenna counterparts. Because of the additional degree of freedom added to the system through the space dimension, one can either attain increasing gains in spectral efficiency or improve the system's error performance by reducing its sensitivity to fading channel conditions. These two aspects are known as

multiplexing and diversity gain, respectively, and the trade-off between them is a 
fundamental problem in the design of multiple antenna system. It is important to note, however, that such gains are achieved under the assumption that the spatial paths from transmit to receive antennas are uncorrelated.

\subsubsection{Multiplexing Gain}

In multiple antenna systems, where the path gains from transmitter to receiver are statistically independent, multiplexing gain can be interpreted as the increase in the system's spectral efficiency, represented as the bit rate per Hertz of bandwidth use. Assuming multiple antennas at the transmitter end, multiplexing gain can be achieved by transmitting different information symbols from each antenna at a given time interval. That is, information is transmitted in parallel effectively taking advantage of the independent spatial channels from transmitter to receiver. For an increasing signal-to-noise ratio (SNR), the multiplexing gain $r$ is expressed as [2]:

$$
r=\lim _{S N R \rightarrow \infty} \frac{R(S N R)}{\log (S N R)}
$$

where $R(S N R)$ is the rate of the transmission code.

\subsubsection{Diversity Gain}

Diversity techniques are based on the premise that by providing multiple copies of a symbol transmitted through independent paths, the probability of all copies experiencing fading decreases. In multiple antenna systems, assuming the antennas at the transmitter are sufficiently separated so as to provide independent fading paths, diversity is achieved by exploiting the spatial dimension of the system. That is, at a given time interval, replicas of a specific information symbol are transmitted from 
each antenna. From this definition, it is clear that a system cannot experience maximal diversity and multiplexing gains at the same time.

From the above description of diversity, one can infer that an increase in diversity leads to a reduction in the error probability, $P_{e}$, in fading channels. This can be illustrated using the following example: given a binary PSK system under fading conditions, the probability of error at high SNR can be approximated as [18]:

$$
P_{e} \approx\left(\frac{1}{4 S N R}\right)^{L}\left(\begin{array}{c}
2 L-1 \\
L
\end{array}\right)
$$

where $L$ is the number statistically independent paths from transmitter to receiver also known as diversity order. If $L=1, P_{e}$ becomes

$$
P_{e} \approx \frac{1}{4} S N R^{-1}
$$

Increasing the diversity order, $L$, to two leads to an error probability of:

$$
P_{e} \approx \frac{3}{16} S N R^{-2}
$$

As it can be seen, from the results in equations (3.3) and (3.4), the probability of error decreases as the $L^{\text {th }}$ power of the SNR [18]. That is, a higher diversity order yields a lower probability of error at a given SNR.

Just like in the case of multiplexing gain, diversity gain, $d$, can be expressed in terms of the received SNR as [2]:

$$
d=-\lim _{S N R \rightarrow \infty} \frac{P_{e}(S N R)}{\log (S N R)}
$$


Since the initial objective of this dissertation is to improve the error rate performance of a PPM communications system using multiple antennas, focus is placed on the implementation of systems using spatial diversity techniques such as space-time coding (STBC).

\subsection{System Model for 2x1 Alamouti Scheme using PPM}

We now explore the system model for a $2 x 1$ polynomial phase modulated (PPM) system using an orthogonal STBC (OSTBC). The overall scheme is presented in

Fig. 3.1. For two transmit antennas and two symbol intervals $(k=\{1,2\})$, the OSTBC transmission matrix based on the Alamouti scheme, given in [3], is:

$$
\boldsymbol{G}(s)=\left(\begin{array}{cc}
s_{1}(t) & s_{2}(t) \\
-s_{2}^{*}(t) & s_{1}^{*}(t)
\end{array}\right)
$$

where $s_{1}(t)$ and $s_{2}(t)$ are two independently generated PPM symbols. Then, given a set of two PPM signals, $\left\{s_{1}(t), s_{2}(t)\right\}$, defined for $k$ symbol intervals as [21]:

$$
s(t)=\exp \left(j\left(\omega_{c} t+\varphi(t)\right)\right.
$$

for $(k-1) T \leq t \leq k T$, the Alamouti scheme, in equation (3.6), means that during the first transmission interval symbols $s_{1}(t)$ and $s_{2}(t)$ are transmitted from antennas 1 and 2 respectively. In the second interval, symbol $-s_{2}^{*}(t)$ is transmitted from antenna 1 and $s_{1}^{*}(t)$ from antenna 2. Based on this, for a single transmission block, the received signal $r_{k}$ at symbol interval $k$ is

$$
r_{k}(t)=\sum_{n=1}^{2} \alpha_{n} e^{j \theta_{n}} G(s)_{k, n}+\eta_{k}(t)
$$

where each coefficient of the $2 \times 2$ transmission matrix $\boldsymbol{G}(s)$ is denoted as $G(s)_{k, n}$. Here we consider the case where the average energy of the symbols transmitted from each 


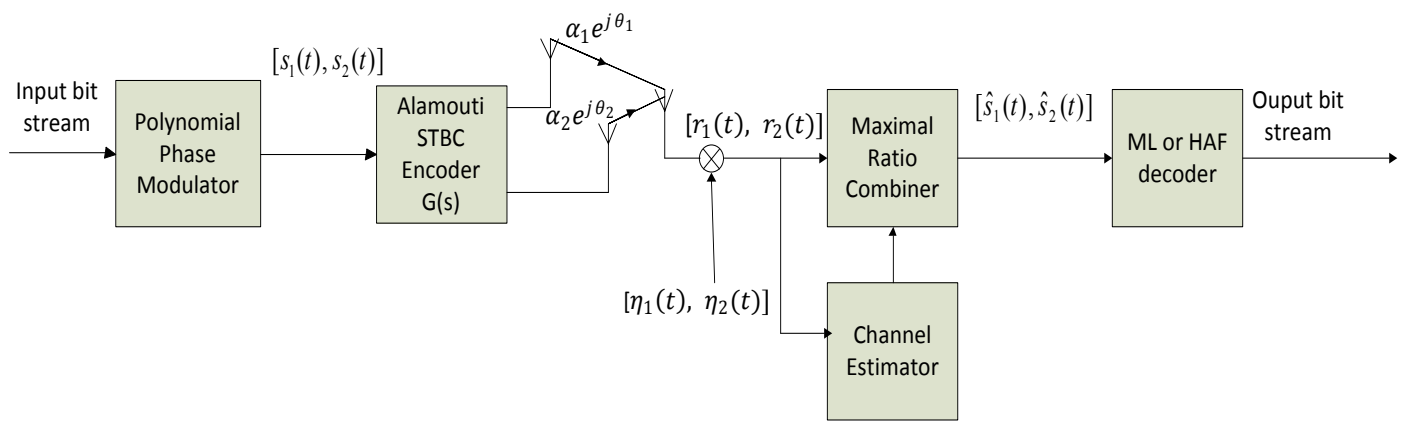

Fig. 3.1. Transmitter and Receiver Model for PPM-OSTBC

antenna has been normalized. Also, the coefficients $\alpha_{n} e^{j \theta_{n}}$ are independent identically distributed (i.i.d) complex Gaussian random variables which represent the components of a $N \times 1$ quasi-static flat fading wireless channel. Finally, $\eta_{k}(t)$ are the components of a $2 \times 1$ vector of independent samples of a zero mean complex Gaussian random variable. Assuming perfect channel knowledge at the receiver and after maximal ratio combining (MRC), an estimate of the transmitted sequence can be obtained from

$$
\begin{aligned}
& \hat{s}_{1}(t)=\left(\alpha_{1}^{2}+\alpha_{2}^{2}\right) s_{1}(t)+\alpha_{1} e^{-j \theta_{1}} \eta_{1}(t)+\alpha_{2} e^{j \theta_{2}} \eta_{2}^{*}(t) \\
& \hat{s}_{2}(t)=\left(\alpha_{1}^{2}+\alpha_{2}^{2}\right) s_{2}(t)-\alpha_{1} e^{j \theta_{1}} \eta_{2}^{*}(t)+\alpha_{2} e^{-j \theta_{2}} \eta_{1}(t) .
\end{aligned}
$$

Since the signals in (3.9) are essentially scaled PPM signals embedded in noise, the transmitted information bits can be recovered by demodulating $\hat{s}_{1}(t)$ and $\hat{s}_{2}(t)$ using either the ML method from equation (2.9) or the lower complexity algorithms such as SML in (2.10), or the HAF illustrated through (2.11) and (2.12). Because of the orthogonality of the Alamouti code, symbol-wise decoding can be performed at the 
receiver and low-complexity algorithms such as S-ML or HAF can be used to recover the transmitted information.

\subsection{Simulation Results}

In this section, the BER performance of the proposed PPM-OSTBC with symbol-wise decoding using Monte-Carlo simulations is evaluated. The system performance was studied when different decoders (ML, S-ML, and HAF) were used for demodulation and under the assumption of normalized average energy per transmitted symbol. For these simulations, we employed systems using linear-binary, $2^{\text {nd }}$ order-binary, and $3^{\text {rd }}$ orderbinary PPM signals. In addition to this, it was assumed that the coefficients of the Rayleigh frequency flat fading channel were constant during one block of code transmission and were known at the receiver.

The BER performance of the OSTBC system for two transmit antennas and one receive antenna, presented in the previous section, with linear-binary PPM is shown in Fig. 3.2. The error rate for a SISO linear-binary PPM and a 2x1 OSTBC system using QPSK are also plotted in this figure. There, the advantage of using multiple antennas at the transmitter end is demonstrated by the obvious difference in error rate performance between the SISO and the OSTBC systems especially at high SNRs, where the diversity gain is more evident. In the figure, it is also observed that the linear-binary PPM system using ML has a similar performance to that using QPSK. This is expected from the results obtained in the SISO case, where it was noted that both systems have the same minimum Euclidean distance for contiguous symbols. On the other hand, when suboptimal decoders are used the BER increases due to propagation errors from high to low order coefficients. 
Fig. 3.3 shows the error rate performances of the $2 \times 1$ OSTBC systems for $2^{\text {nd }}$ orderbinary PPM using an ML demodulator. The BER of a 2x1 OSTBC using 8PSK is also shown for comparison. It is again observed that PPM-OSTBC systems exhibit lower error rates than the 8PSK-OSTBC one. Specifically, at an error rate of $10^{-1}$, the PPM-OSTBC system using ML requires approximately $1 \mathrm{~dB}$ less power than the 8PSK-OSTBC system. Also, as observed in the SISO case, as the polynomial order increases, the error rate performance of the PPM-based system degrades when sub-optimal decoders are used for demodulation.

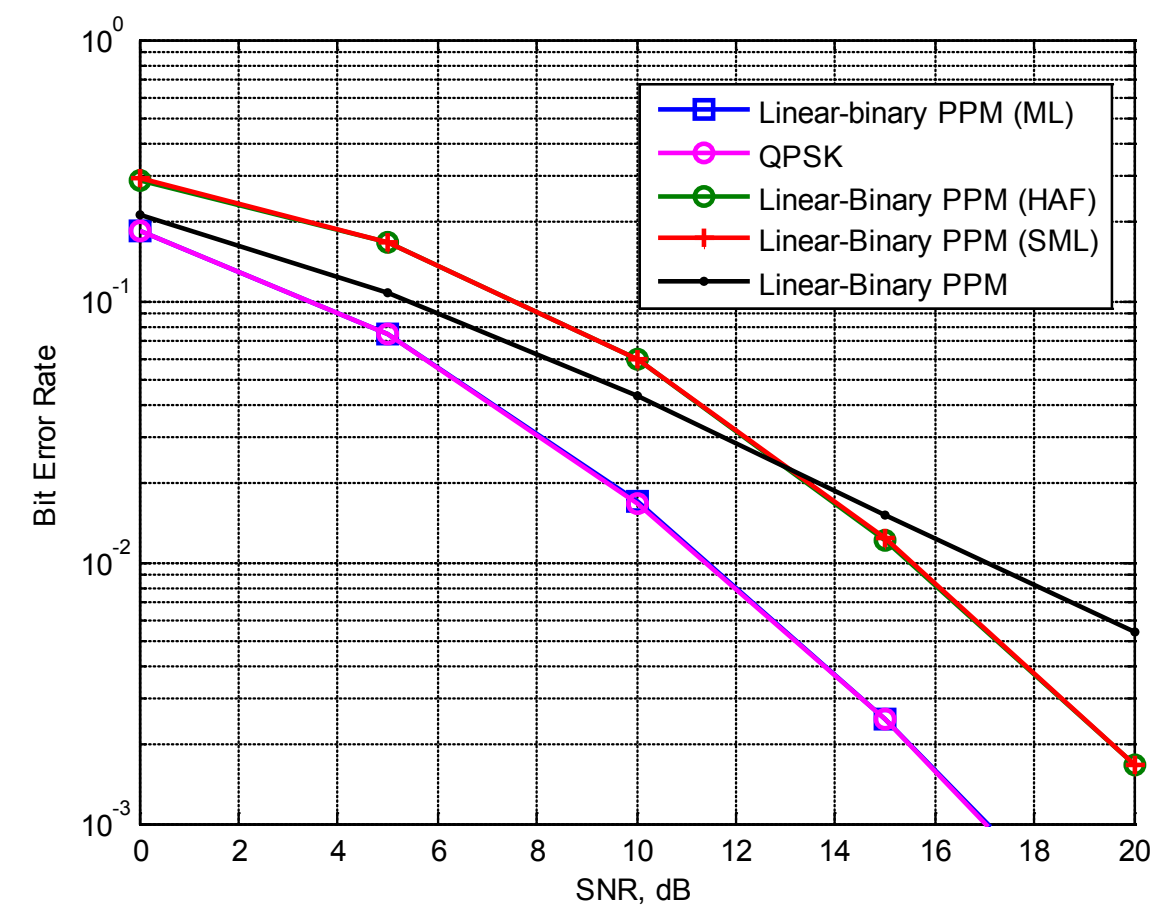

Fig. 3.2 BER Performance Comparison of SISO Linear-binary PPM, Linear-Binary PPM-OSTBC and QPSK-OSTBC

In Fig. 3.4 the comparison between the BER curves for the $3^{\text {rd }}$ order-binary PPMOSTBC and the 16PSK-OSTBC systems is presented. From the figure, it is evident that 
the PPM system outperforms the 16PSK system by more than $2 \mathrm{~dB}$ at an error rate of $10^{-1}$. Again, the observed results are a direct consequence of the higher Euclidean distances between $3^{\text {rd }}$ order-binary PPM symbols.

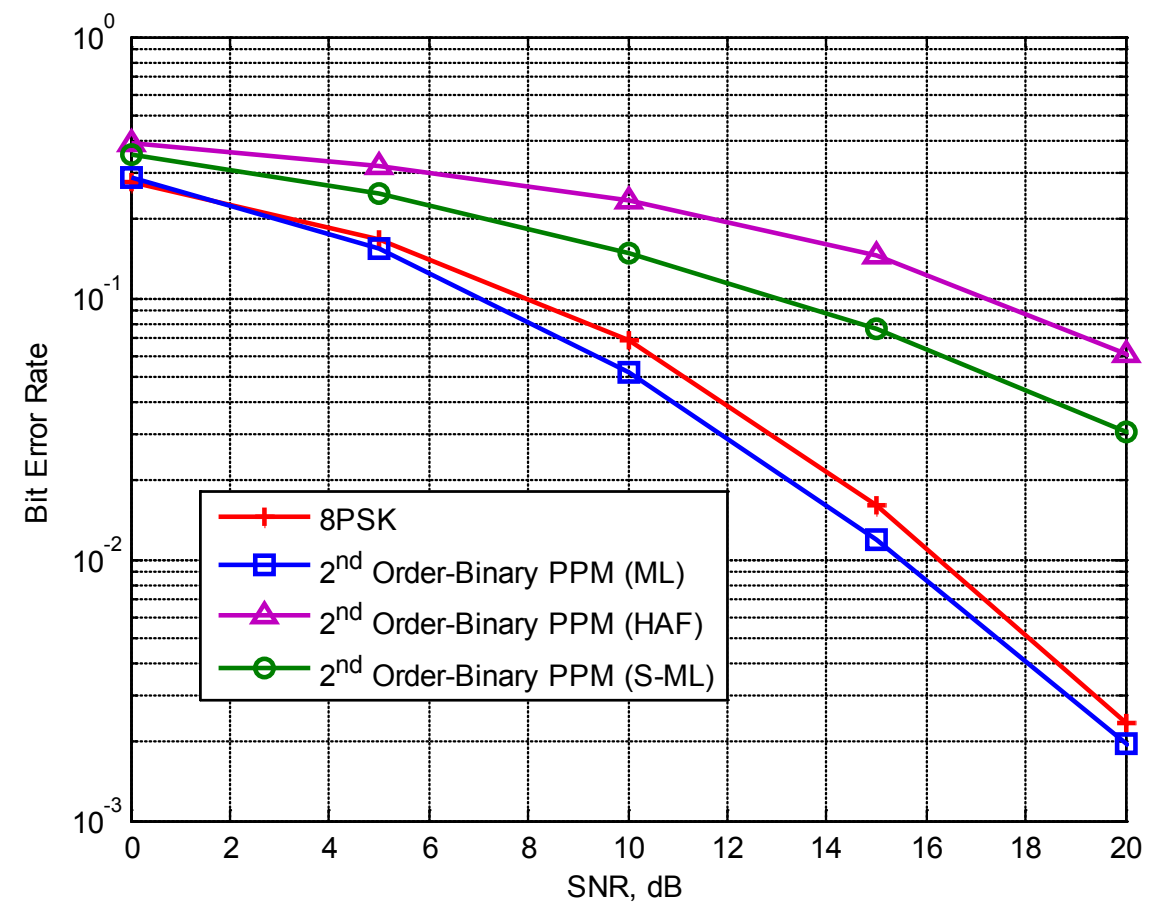

Fig. 3.3 BER Performance Comparison of $2^{\text {nd }}$ Order-Binary PPM-OSTBC and 8PSKOSTBC

\subsection{Conclusions}

In this chapter, a study on the performance of PPM signals in systems with two transmit antennas was presented. Through Monte-Carlo simulations, it was demonstrated that such systems exhibit an improvement in error rate performance with respect to the single antenna case. Using the Alamouti scheme allows for the use of sub-optimal decoding algorithms that alleviate the complexity of the system. Nevertheless, it was observed that such techniques also lead to increased error rates. In addition to this, just as in the SISO 
case, the proposed OSTBC systems using PPM signals and ML showed a better error rate performance than their PSK counterparts, especially when high transmission rates were required. This error rate performance improvement translates into lower energy requirements for the mobile device and therefore larger battery life.

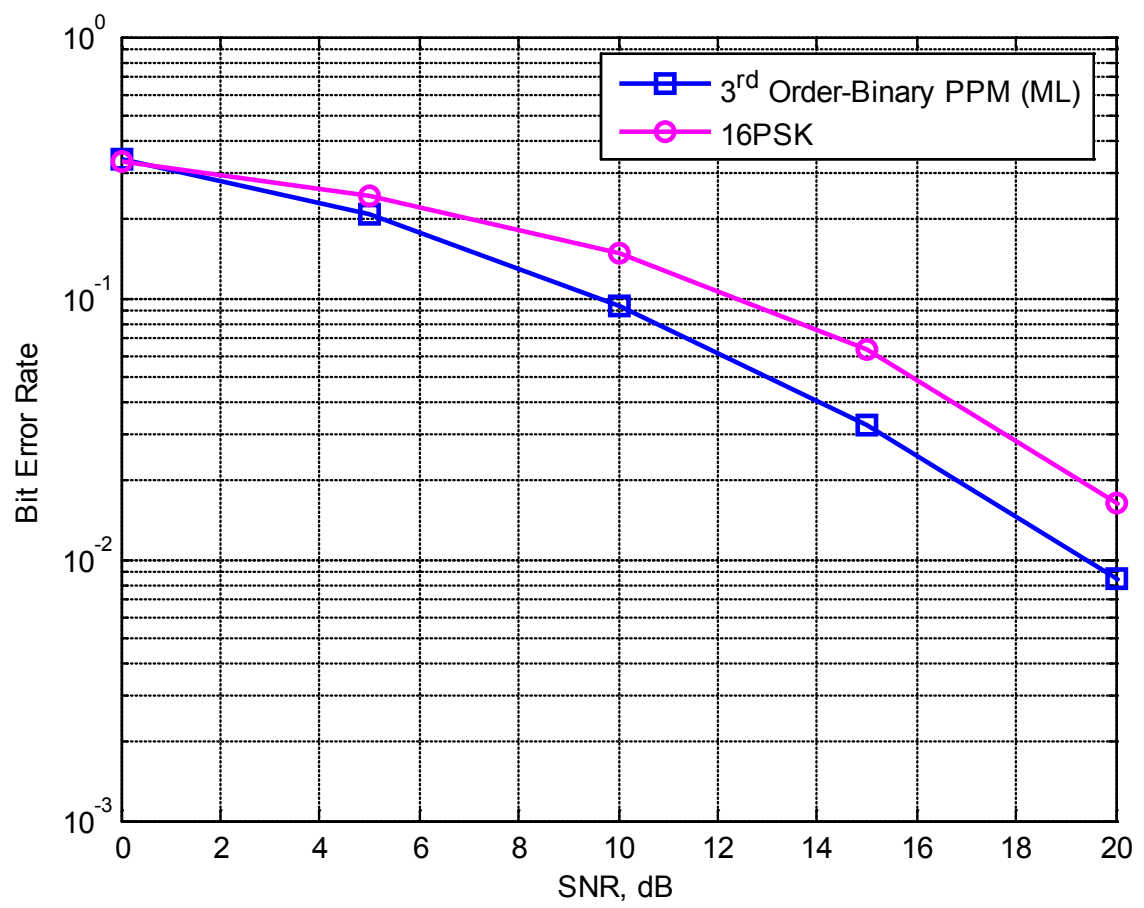

Fig. 3.4 BER Performance Comparison of $3^{\text {rd }}$ Order-Binary PPM-OSTBC and 16PSKOSTBC 


\section{CHAPTER 4}

\section{FULL DIVERSITY QOSTBC FOR PPM SIGNALS}

In Chapter 3, it was shown that increasing the number of transmit antennas might lead to a considerable improvement in error rate performance given that the paths from these antennas to the receiver are independent. To take advantage of the potential diversity gain, Dam et al [9] proposed a space-time module structure for PPM signals. The proposed module structure allows for the simple design of real orthogonal space-time codes for more than two transmit antennas because the encoding is performed on the phase coefficients. Nevertheless, in [9], it is also shown that the error rate performance of such structure is worse than conventional modulation formats at high signal-to-noise ratios (SNR). The reason behind this decline in performance is that by encoding only the phase coefficients, the module structure does not ensure that the transmitted signals would in fact provide full transmit diversity. In addition to this, the system in [9] requires significant changes from current MIMO systems used for conventional modulation formats, which would make system upgrades and/or adaptation more difficult and expensive.

In this chapter, in contrast with [9], we design full-diversity STBC PPM systems for four transmit antennas by directly encoding the PPM modulated signal. However, since full transmission rate cannot be accomplished with complex orthogonal STBCs when more than two transmit antennas are used, the quasi-orthogonal STBC proposed by Jafarkhani in [4] is used to encode optimally rotated PPM constellations. Here, we obtain the optimal rotation angle for binary PPM signal constellations that guarantee maximum diversity. Then, through Monte Carlo simulations, it is shown that rotating the 
constellation by the optimal angle yields an improved bit error rate (BER) performance compared with non-rotated PPM constellations. In these simulations, it is also shown that the proposed systems perform better in terms of error rate than conventional systems using PSK modulation.

This chapter is organized as follows. In section 4.1, the system and polynomial phase modulation (PPM) signal model are presented. In section 4.2, the Jafarkhani scheme for quasi-orthogonal space time codes is described, as well as, its condition for full diversity. In section 4.4, the optimal constellations for the proposed modulation format using different polynomial phase orders are obtained. Simulation of the error rate performance of a QOSTBC using different PPM constellations is presented in section 4.5. Concluding remarks are presented in section 4.6.

\subsection{System and Signal Model}

For a system with $N$ transmit and $M$ receive antennas, the received signal $r_{k, m}$ at time instant $k$ and antenna $m$ is given by

$$
r_{k, m}=\sum_{n=1}^{N} h_{n, m} C_{k, n}+\eta_{k, m}
$$

where $1 \leq k \leq T$ and each coefficient of the $T \times N$ transmission matrix $C$ is denoted as $C_{k, n}$. The coefficients $h_{n, m}$ are independent identically distributed (i.i.d) complex Gaussian random variables which represent the components of an $N \times M$ quasi-static flat fading wireless channel. Finally, $\eta_{k, m}$ are the components of a $T \times M$ matrix of independent samples of a zero mean complex Gaussian random variable. 
In this chapter, a wireless communications system with four transmit antennas and one receive antenna is considered for simplicity; however, the system can be easily extended to more than one receive antenna.

The modulation format used here is known as polynomial phase modulation and the modulated signal $s(t)$ for a symbol period $T_{0}$ is

$$
s(t)=\operatorname{Aexp}\left(j\left(\omega_{c} t+\varphi(t)\right)\right.
$$

where $A$ is the signal amplitude and is assumed to be unity, $0 \leq t \leq T_{0}$, and

$$
\varphi(t)=a_{M}\left(t / T_{0}\right)^{M}+a_{M-1}\left(t / T_{0}\right)^{M-1}+\ldots+a_{1}\left(t / T_{0}\right)+a_{0}
$$

is the time-varying phase whose phase coefficients are chosen as $a_{i} \in\{ \pm \pi / 2\}$.

The system block diagram is illustrated in Fig. 4.1. In the diagram, it is shown that the input binary stream is mapped onto a sequence of PPM symbols. Each set of four symbols $\left(s_{1}, s_{2}, s_{3}, s_{4}\right)$ is then transformed by the $T \times N$ transmission matrix $C$. At the receiver end, the signal is decoded through pair-wise maximum likelihood (ML) decoding to recover the original bit stream.

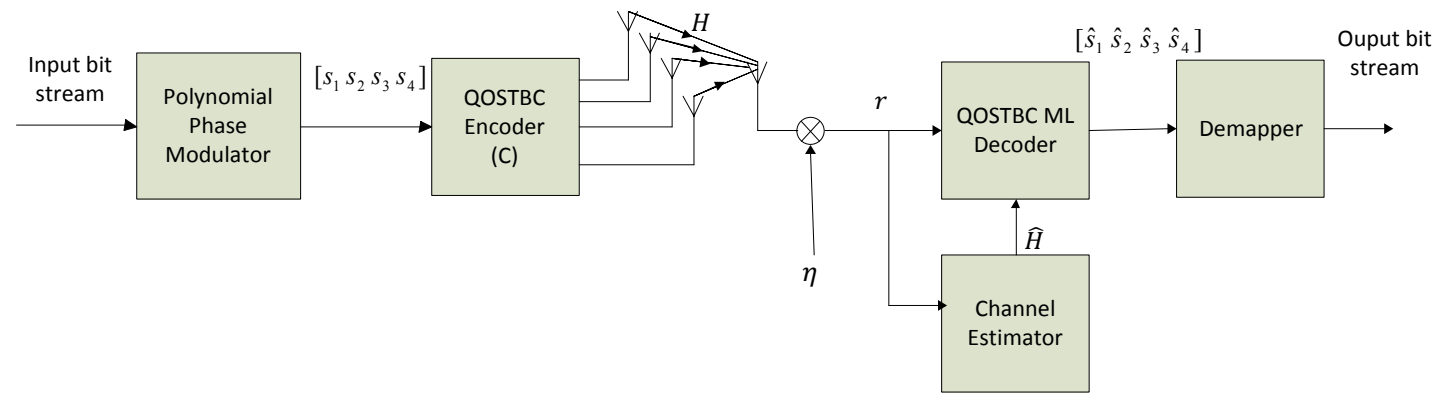

Fig. 4.1System Block Diagram 


\subsection{STBC from Quasi-Orthogonal Design}

Since full transmission rate cannot be accomplished with complex orthogonal STBCs when more than two transmit antennas are used, the quasi-orthogonal STBC proposed by Jafarkhani in [4] is employed.

As mentioned in chapter 2, for two transmit antennas, Alamouti [3] proposed the following full rate orthogonal STBC for complex signal constellations.

$$
\boldsymbol{G}\left(s_{1}, s_{2}\right)=\left(\begin{array}{cc}
s_{1} & s_{2} \\
-s_{2}^{*} & s_{1}^{*}
\end{array}\right)
$$

Then, for four transmit antennas Jafarkhani expanded the Alamouti scheme to

$$
\boldsymbol{C}=\left(\begin{array}{cc}
\boldsymbol{G}\left(s_{1}, s_{2}\right) & \boldsymbol{G}\left(s_{3}, s_{4}\right) \\
-\boldsymbol{G}^{*}\left(s_{3}, s_{4}\right) & \boldsymbol{G}^{*}\left(s_{1}, s_{2}\right)
\end{array}\right)
$$

From (4.3) and (4.4), it follows that

$$
\boldsymbol{C}=\left(\begin{array}{rrrr}
S_{1} & S_{2} & S_{3} & S_{4} \\
-S_{2}^{*} & S_{1}^{*} & -S_{4}^{*} & S_{3}^{*} \\
-S_{3}^{*} & -S_{4}^{*} & S_{1}^{*} & S_{2}^{*} \\
S_{4} & -S_{3} & -S_{2} & S_{1}
\end{array}\right)
$$

The block code shown in equation (4.5) clearly achieves full rate as one symbol is transmitted per time interval. Nevertheleson of the code orthogonality requirement, decoding complexity increases with respect to that of orthogonal codes because only pairwise decoding is possible. That is, based on ML decoding, the transmitted symbols can be estimated as the symbols that minimize the decision metrics $f_{14}\left(s_{1}, s_{4}\right)$ and $f_{23}\left(s_{2}, s_{3}\right)$ over all pairs of symbols as [12]: 


$$
\begin{aligned}
f_{14}\left(s_{1}, s_{4}\right)= & \left(\left|s_{1}\right|^{2}+\left|s_{4}\right|^{2}\right)\left(\sum_{n=1}^{4}\left|h_{n}\right|^{2}\right) \\
& +2 \mathfrak{R}\left\{\left(-h_{1} r_{1}^{*}-h_{2}^{*} r_{2}-h_{3}^{*} r_{3}-h_{4} r_{4}^{*}\right) s_{1}\right. \\
& \left.+\left(-h_{4} r_{1}^{*}+h_{3}^{*} r_{2}+h_{2}^{*} r_{3}-h_{1} r_{4}^{*}\right) s_{4}\right\} \\
& +4 \Re\left\{h_{1} h_{4}^{*}-h_{2}^{*} h_{3}\right\} \Re\left\{s_{1} s_{4}^{*}\right\}
\end{aligned}
$$

and

$$
\begin{aligned}
f_{23}\left(s_{2}, s_{3}\right)= & \left(\left|s_{2}\right|^{2}+\left|s_{3}\right|^{2}\right)\left(\sum_{n=1}^{4}\left|h_{n}\right|^{2}\right) \\
& +2 \Re\left\{\left(-h_{2} r_{1}^{*}+h_{1}^{*} r_{2}-h_{4}^{*} r_{3}+h_{3} r_{4}^{*}\right) s_{2}\right. \\
& \left.+\left(-h_{3} r_{1}^{*}-h_{4}^{*} r_{2}+h_{1}^{*} r_{3}+h_{2} r_{4}^{*}\right) s_{3}\right\} \\
& +4 \Re\left\{h_{2} h_{3}^{*}-h_{1}^{*} h_{4}\right\} \Re\left\{s_{2} s_{3}^{*}\right\}
\end{aligned}
$$

\subsection{Constellation Rotation for Full Diversity QOSTBC Systems}

Assuming an ML decoder is being used, for a pair of transmitted codewords

$$
\boldsymbol{C}=\boldsymbol{C}\left(s_{1}, s_{2}, s_{3}, s_{4}\right) \text { and } \boldsymbol{C}^{\prime}=\boldsymbol{C}\left(s_{1}^{\prime}, s_{2}^{\prime}, s_{3}^{\prime}, s_{4}^{\prime}\right),
$$

the upper bound of the probability of wrongfully decoding a transmitted word $\boldsymbol{C}$ as $\boldsymbol{C}^{\prime}$, known as the pairwise error probability (PEP), for one receive antenna is [20]

$$
P\left(\boldsymbol{C} \rightarrow \boldsymbol{C}^{\prime}\right) \leq \frac{4^{r}}{\left(\prod_{n=1}^{r} \lambda_{n}\right) \gamma^{r}}
$$


where $\gamma$ is the received SNR and $r$ is the rank of the difference matrix $\boldsymbol{D}\left(\boldsymbol{C}, \boldsymbol{C}^{\prime}\right)=$ $\left(\boldsymbol{C}^{\prime}-\boldsymbol{C}\right)$. Also the parameters $\lambda_{n}$ represent the eigenvalues of the matrix $\boldsymbol{A}\left(\boldsymbol{C}, \boldsymbol{C}^{\prime}\right)=$ $\boldsymbol{D}\left(\boldsymbol{C}, \boldsymbol{C}^{\prime}\right)^{H} \boldsymbol{D}\left(\boldsymbol{C}, \boldsymbol{C}^{\prime}\right)$ where and $\boldsymbol{D}(\cdot)^{H}$ is the Hermitian of $\boldsymbol{D}(\cdot)$.

From equation (4.9), it can be seen that in order to reduce the pairwise error probability one should design space-time codes to ensure that the rank of the difference matrix and the minimum value of the product $\prod_{n=1}^{r} \lambda_{n}$ over all possible codeword pairs are as large as possible. These two conditions are known as the diversity and the product criterion, respectively [20]. Notice that the diversity criterion is also equivalent to ensuring that the coding gain distance (CGD) defined as

$$
C G D=\operatorname{det}\left[\boldsymbol{D}\left(\boldsymbol{C}, \boldsymbol{C}^{\prime}\right)^{H} \boldsymbol{D}\left(\boldsymbol{C}, \boldsymbol{C}^{\prime}\right)\right]
$$

is non-zero for any possible pair of distinct codewords.

For the quasi-orthogonal STBC in (4.5), this condition cannot be met when all symbols in each codeword are chosen from the same constellation; therefore full diversity cannot be attained. So in addition to receiver complexity, the resulting lower diversity is another important limitation of QOSTBCs. This ultimately causes a decrease in BER performance at high SNRs compared to orthogonal STBCs. In order to achieve full diversity it is necessary to ensure the CGD in equation (4.10) is not zero; therefore, a way to address this issue is to choose symbols $s_{1}$ and $s_{2}$ from the regular constellation and $s_{3}$ and $s_{4}$ from a different one. It has been proven in [5] and [12] that this simple scheme guarantees the system is full-diversity; however, in order to truly take advantage of the multiple transmit antennas, the product criterion must also be met. Consequently, if matrix $\boldsymbol{A}\left(\boldsymbol{C}, \boldsymbol{C}^{\prime}\right)$ is full rank, then the product criterion is satisfied if the second 
constellation is chosen such that the minimum CGD is maximized. For a signal constellation $\mathcal{A}$, this optimization problem is described in [12] as

$$
C G D_{\min }(\phi(t))=\max _{\left(s_{1}, \tilde{s}_{3}\right) \neq\left(s_{1}^{\prime}, \tilde{s}_{3}^{\prime}\right)}\left|\left(s_{1}-s_{1}^{\prime}\right)^{2}-\left(\tilde{s}_{3}-\tilde{s}_{3}^{\prime}\right)^{2}\right|^{4}
$$

where $\mathrm{s}_{1} \in \mathcal{A}$ and $\widetilde{\mathrm{s}_{3}} \in \mathrm{e}^{\mathrm{j} \emptyset(\mathrm{t})} \mathcal{A}$.

If $d_{\min }=\min \left(\left|s_{1}-s_{1}^{\prime}\right|\right)$, then the upper bound of the minimum CGD can be expressed as [12]

$$
C G D_{\min }(\phi(t)) \leq d_{\min }^{8}
$$

That is, if a modulation type has a $C G D_{\min }$ equal to $d_{m i n}^{8}$, it is said that this modulation format has achieved the maximum minimum $C G D$ possible. Thus, the rotation angle at which this happens is considered to be the optimum rotation angle in terms of providing the highest minimum $C G D$ possible.

\subsection{Optimal PPM Constellations}

As mentioned in the previous section, full diversity can be achieved if symbols $\widetilde{s_{3}}$ and $\widetilde{s_{4}}$ are chosen from a constellation rotated with respect to the one used for symbols $S_{1}$ and $s_{2}$. The optimization problem is thus, to select the proper rotation angle function, $\varnothing(t)$, that maximizes the minimum CGD defined in equation (4.11).

For the case of linear binary polynomial phase modulation, where the phase coefficients are chosen from $a_{i} \in\{ \pm \pi / 2\}$, the rotation angle function considered is of the form

$$
\varnothing(t)=\emptyset_{1}\left(\frac{t}{T_{0}}\right)+\emptyset_{0}
$$


where $\emptyset_{1}$ and $\emptyset_{0}$ are the rotation angles for the first-order and constant phase coefficients respectively and can be chosen from the range $0 \leq \emptyset_{i} \leq \pi / 2$. Based on this, the surface plot of the minimum CGD for linear-binary PPM using different rotation angles was generated and it is shown in Fig. 4.2. In this figure, it can be seen that the rotation angle function that maximizes the minimum CGD is not unique. In fact, as long as the rotation angle for the constant phase coefficient is approximately higher than $\pi / 6$, the system achieves the upper bound of the $\mathrm{CGD}_{\min }$, shown in equation (4.12), regardless of the value of $\emptyset_{1}$. Therefore, it is only necessary to appropriately choose the value of $\emptyset_{0}$.

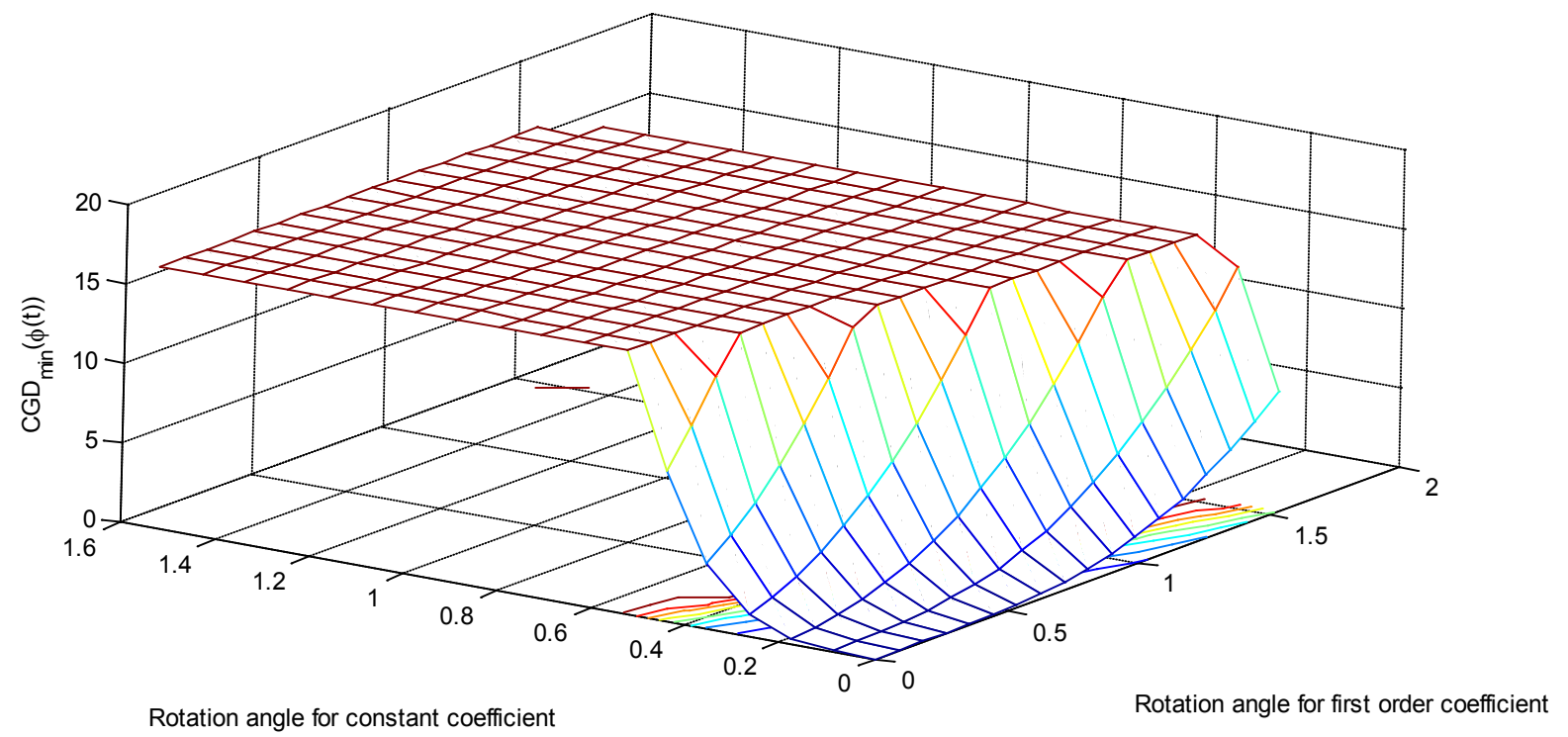

Fig. 4.2 CGD $D_{\min }(\phi(t))$ for Rotations on the Constant and First-Order Coefficients

In order to have a better idea of what rotation angle yields the best error rate performance and thus the most energy efficient system, based on the cost functions in equations (4.6) and (4.7), a new metric known as channel interference coefficient (CIC) is introduced. The CIC is the coefficient that multiplies the channel components in the fourth term to 
the right of equations (4.6) and (4.7). Namely, CIC $=\left|\mathfrak{R}\left\{s_{1} \tilde{s}_{4}^{*}\right\}\right|=\left|\mathfrak{R}\left\{s_{2} \tilde{s}_{3}^{*}\right\}\right|$ for $s_{1}$, $s_{2} \in \mathcal{A}$ and $\tilde{s}_{3}, \tilde{s}_{4} \in e^{j \phi} \mathcal{A}$. It can be observed that as the value of this factor increases, the effect of the channel interference on the cost functions, (4.6) and (4.7), grows, leading to an increase in error rate. This means that this parameter has a direct effect on the transmitted sequence estimation process; therefore, one should choose the rotation angle so that the maximum CIC is minimized over all possible symbol pairs $s_{1}, \tilde{S}_{4}$. That is, one should find the angle, $\phi$, that minimizes

$$
C I C_{\max }(\phi)=\min _{s_{1}, \tilde{s}_{4}}\left(\left|\Re\left\{s_{1} \tilde{s}_{4}^{*}\right\}\right|\right) .
$$

In Fig. 4.3, the plots for the maximum $C G D_{\min }$ and the minimum $C I C_{\max }$ are shown for different rotation angles for a QOSTBC system using a linear-binary PPM. Notice that the figure is only shown for the case when the rotation is applied to $a_{0}$. From the figure, it is clear that the value that optimizes both metrics is $\emptyset=\emptyset_{0}=1$.

Let us now consider the case in which the constellation consists of $2^{\text {nd }}$ order-binary PPM signals where, again, the phase coefficients are selected from $a_{i} \in\{ \pm \pi / 2\}$. Just as in the case of the linear-binary PPM, we optimize (4.11) and (4.14) numerically for the case where the rotation is applied to the constant phase coefficient. Based on the resulting curves for the maximum $C G D_{\min }$ and the minimum $C I C_{\max }$, it can be seen that the optimal rotation angle is $\emptyset=\emptyset_{0}=1.4$.

Finally, the optimal rotation angle for $3^{\text {rd }}$ order-binary PPM was obtained using the same numerical procedure described for the previous lower order PPM signals. Fig. 4.5 shows the curves for the maximum $C G D_{\min }$ and the minimum $C I C_{\max }$ as functions of the 
rotation angle $\phi$. In the $C G D_{\min }$ curve one can observe a global maximum at approximately 0.6 and a local maximum at $\pi / 2$. The $C I C_{\max }$ curve, on the other hand, has only a global minimum at $\pi / 2$. Since the peaks of the $C G D_{\min }$ curve are relatively close in magnitude, the rotation angle that provides the best possible error rate performance is $\pi / 2$ as it yields a significantly lower $C I C_{\max }$ than 0.6 . It must be observed, however, that the improvement in error rate performance for this order of modulation is not as significant as in the lower order cases, especially at low SNRs. The reason behind this is that even at the optimal rotation angle, the minimum CGD is not significantly larger than for all other rotation angles.
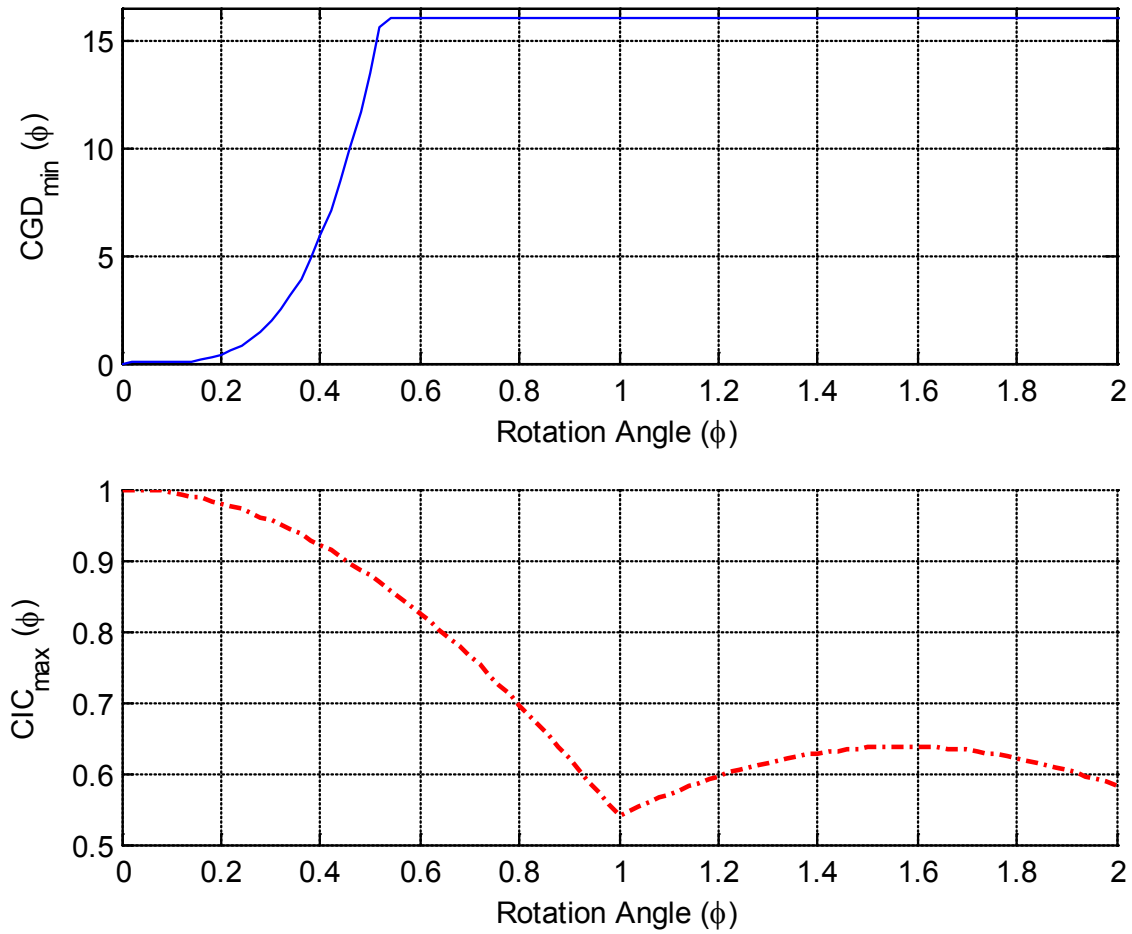

Fig. 4.3CGD $D_{\min }$ and $C I C_{\max }$ vs. Rotation Angle for Linear-binary PPM 

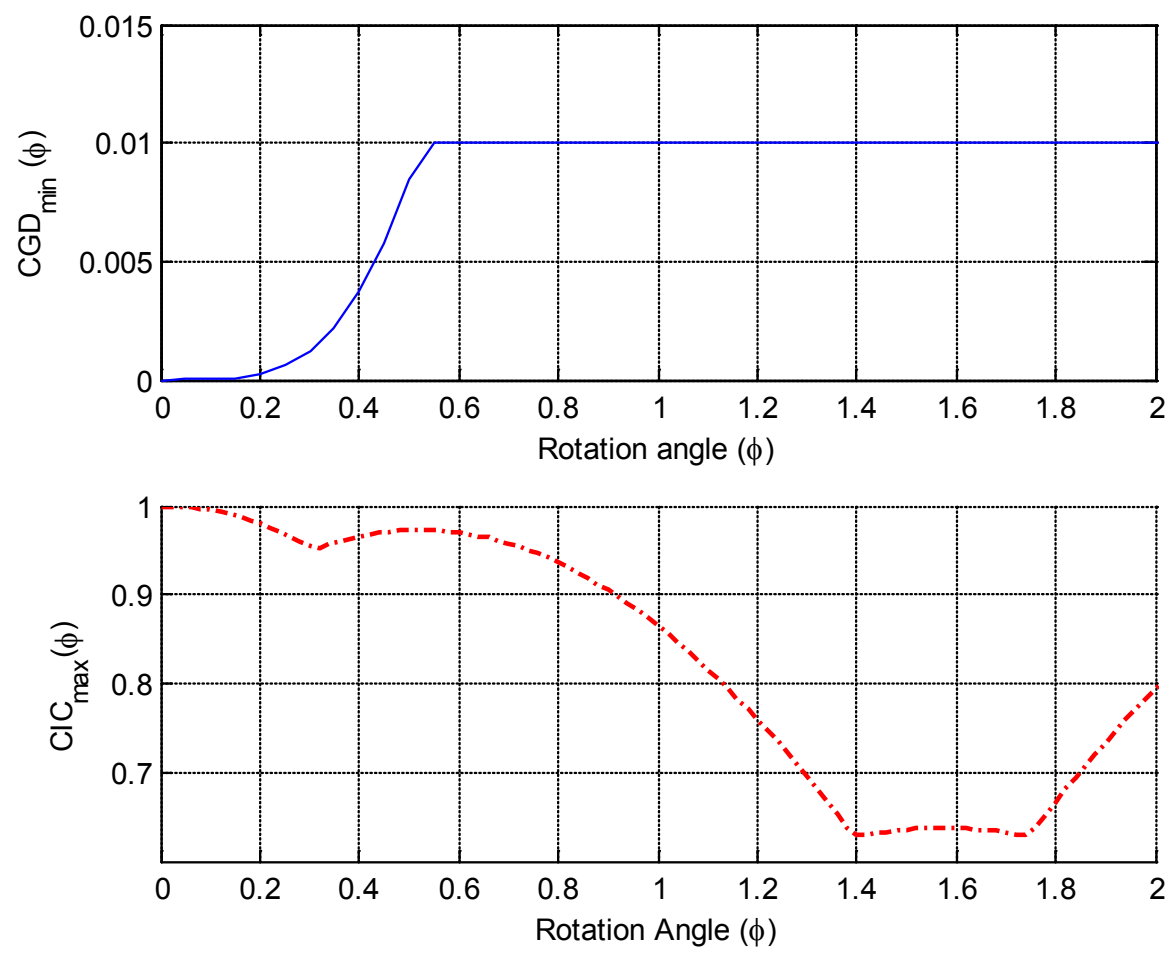

Fig. 4.4CGD $D_{\min }$ and $C I C_{\max }$ vs. Rotation Angle for $2^{\text {nd }}$ Order-Binary PPM
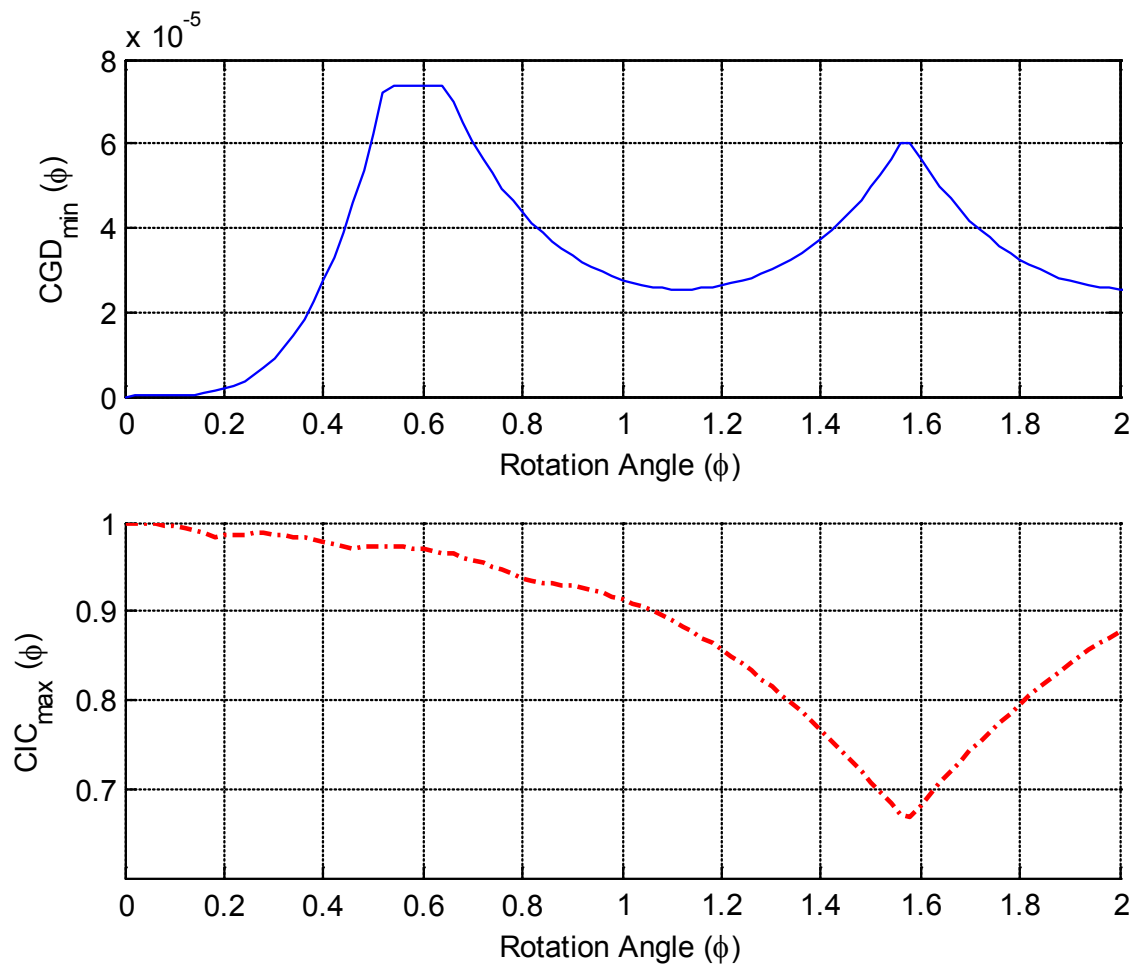

Fig. 4.5 $C G D_{\min }$ and $C I C_{\max }$ vs. Rotation Angle for $3^{\text {nd }}$ Order-Binary PPM 
The results for the above PPM signal formats are summarized on Table 4.1 and compared to M-ary PSK constellations with similar spectral efficiency. At this point, it is important to note that the minimum coding gain distance comparison in Table 4.1 is just to show that, when rotated by the optimal angle, PPM signals achieve a $C G D_{\min }$ close to the upper bound given by $d_{\text {min }}^{8}$, which is not the case for PSK signals. However, this is not an absolute measure of the system's BER performance since it only takes into consideration the coding gain distance among the closest codeword pairs. In order to properly evaluate the error probability, one should take into account the CGD among all possible codeword pairs. As a way of validating these results, in the following section, we evaluate the error rates for QOSTBCs using linear, $2^{\text {nd }}$, and $3^{\text {rd }}$ order binary PPM signals through Monte Carlo simulations and compare them to those using M-ary PSK constellations.

Table 4.1Comparison of $C G D_{\min }$ and $C I C_{\max }$ values for PPM and PSK Constellations

\begin{tabular}{|c|c|c|c|c|c|c|}
\hline & $\begin{array}{c}\text { Linear } \\
\text { PPM }\end{array}$ & QPSK & $\begin{array}{c}\mathbf{2}^{\text {nd }} \text { Order } \\
\text { PPM }\end{array}$ & 8PSK & $\begin{array}{c}3^{\text {rd }} \text { Order } \\
\text { PPM }\end{array}$ & 16PSK \\
\hline $\boldsymbol{\phi}$ & 1 & $\pi / 4$ & 1.4 & $\pi / 8$ & $\pi / 2$ & $\pi / 16$ \\
\hline CGD $_{\min }$ & 16 & 16 & 0.0100 & 0.0404 & $6.20 \mathrm{e}-5$ & $1.24 \mathrm{e}-5$ \\
\hline $\boldsymbol{d}_{\min }^{\mathbf{8}}$ & 16 & 16 & 0.0100 & 0.1178 & $7.39 \mathrm{e}-5$ & $5.37 \mathrm{e}-4$ \\
\hline $\mathbf{C I C}_{\max }$ & 0.5403 & 0.7071 & 0.6287 & 0.9239 & 0.6655 & 0.9808 \\
\hline
\end{tabular}




\subsection{Simulation Results and Discussion}

In the following Monte Carlo simulations, the BER performance of PPM using the Jafarkhani [4] QOSTBC for systems with four antennas at the transmitter and one antenna at the receiver was studied. For these simulations, we used systems with Gray encoded linear, $2^{\text {nd }}$, and $3^{\text {rd }}$ order-binary PPM constellations. We also assumed that the coefficients of the Rayleigh flat fading channel were constant during one block of code transmission and were perfectly known at the receiver.

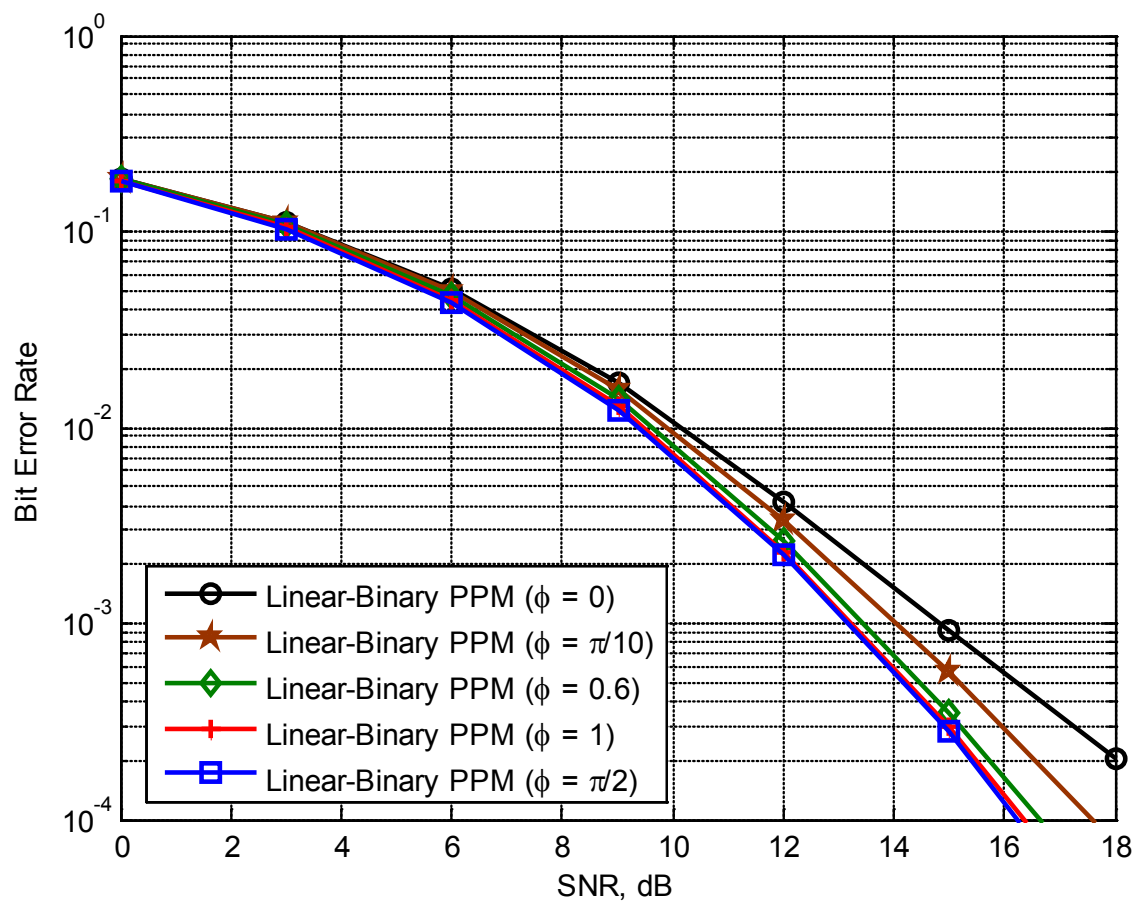

Fig. 4.6 BER Performance of Linear-Binary PPM with QOSTBC for Different Rotation Angles 


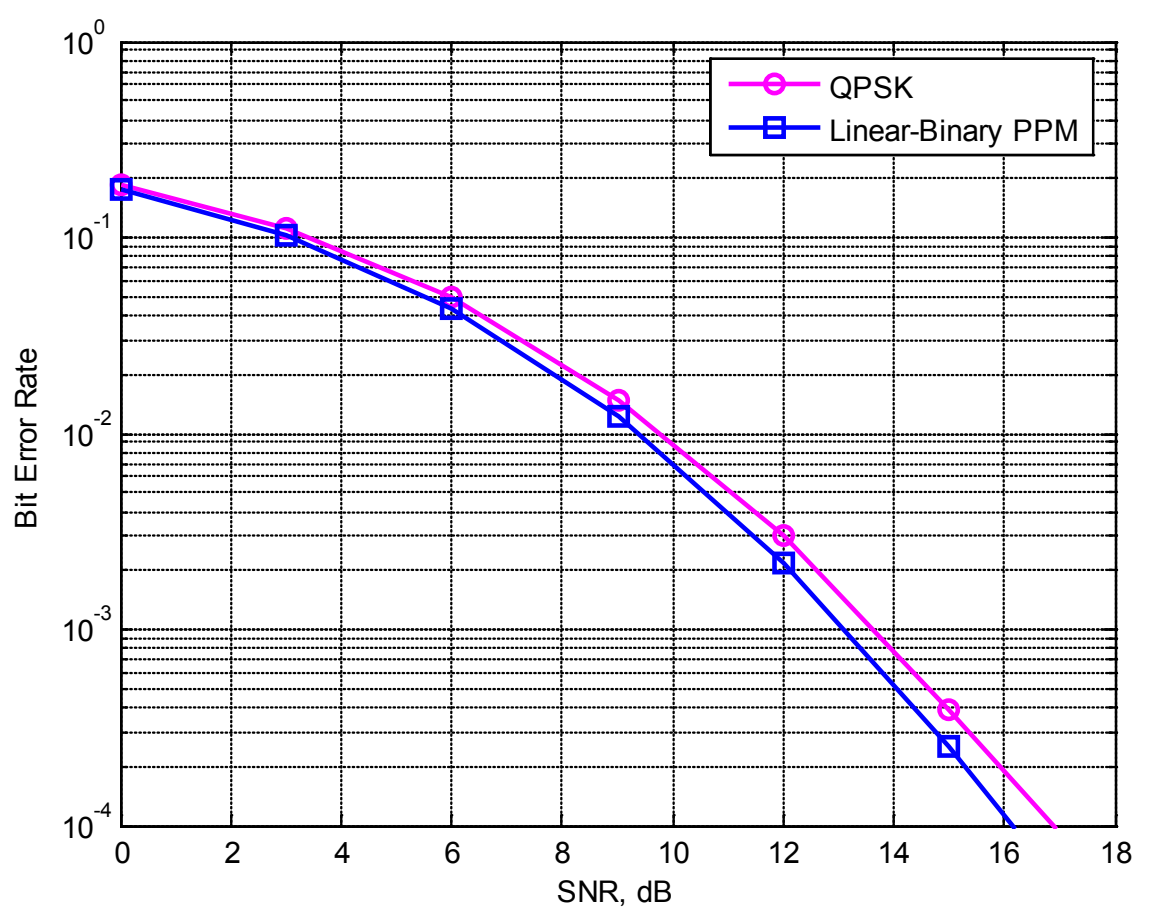

Fig. 4.7 BER Performance for QOSTBC using Linear- Binary PPM and QPSK

In Fig. 4.6, the error rate for a QOSTBC system with a linear binary PPM constellation using different rotation angles is shown. There, it can be seen that the systems with rotation angles of $\pi / 2$ and 1 display a higher diversity order than for any other rotation angle. Specifically, we can notice that the system using the non-rotated constellation has the highest BER for high values of signal-to-noise ratio (SNR). Also, observe that even though $\phi=0.6$ yields the maximum $C G D_{\min }$, the BER is higher than for $\phi=\pi / 2$ because at 0.6 the system doesn't achieve the minimum $C I C_{\max }$, as shown in Fig. 4.3. This is evidence of the need for optimizing both metrics, $C I C_{\max }$ and $C G D_{\min }$, in order to obtain the best BER performance. Next, in Fig. 4.7, the performance of the proposed full diversity QOSTBC using linear-binary PPM is compared to that of a QOSTBC system using QPSK. The error rate in Rayleigh fading channels is lower for linear PPM than for 
QPSK and, as shown in the figure, the proposed system clearly outperforms the system using QPSK in terms of their error rate performance. We can see that at an error rate of $10^{-4}$, the SNR gap between both modulation schemes is about $1 \mathrm{~dB}$ making the linearbinary PPM system a more power efficient alternative than the QOSTBC using QPSK. Notice that even though both systems achieve the same minimum CGD, the scheme with the linear-binary PPM signals achieves a lower $C I C_{\max }$ over all other codeword pairs. From the figure, it can be observed that this ultimately yields a better error rate performance.

The simulated BER performance of $2^{\text {nd }}$ order-binary PPM for different rotation angles is presented in Fig. 4.8. As expected from the discussion in the previous section, the rotation angles 1.4 and $\pi / 2$, yield the highest minimum CGD and the lowest maximum $\mathrm{CIC}$ and thus, the lowest error rate. The proposed full diversity PPM-QOSTBC scheme was then compared with one employing an optimally rotated 8PSK constellation. Again, it can be observed that at an error rate of $10^{-2}$, the system using 8PSK requires approximately $1 \mathrm{~dB}$ more power than the proposed PPM system. However, as the SNR increases the BER gap between both systems decreases because the PSK-based system has a higher $C G D_{\min }$ than the one of the PPM system as shown in Table 4.1.

Fig. 4.10 shows the BER curves for QOSTBC systems using $3^{\text {rd }}$ order-binary PPM with different rotation angles. From the figure, however, it is difficult to distinguish which rotation angle yields the best error rate performance. For this reason the error rates for different rotation angles are also summarized in Table 4.2. It can be seen that for a rotation angle $\phi=\pi / 2$ the QOSTBC system achieves the best BER performance as the error rate is lower than for any other angle at a specific SNR; thus, a more power efficient 
system is obtained. In consequence, based on this and the previous results for lower order binary PPM signals, where the phase coefficients are chosen from $a_{i} \in\{ \pm \pi / 2\}$, it can be conjectured that, for any polynomial order, PPM signals of this family a convenient rotation angle is $\phi=\pi / 2$.

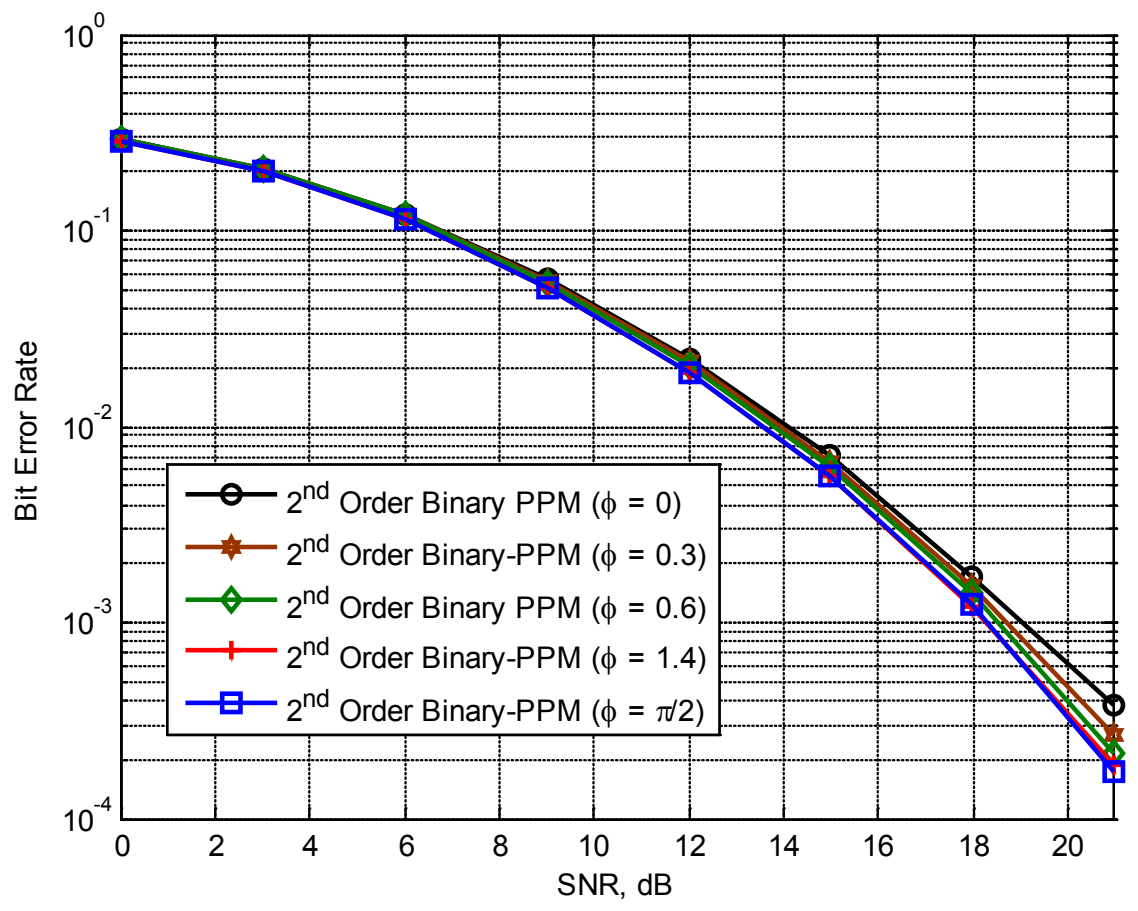

Fig. 4.8 BER Performance for QOSTBC using $2^{\text {nd }}$ Order-Binary PPM for Different Rotation Angles

Lastly, in Fig. 4.11 the performance of a full diversity $3^{\text {rd }}$ order-binary PPM QOSTBC system is compared to that of a similar system using 16PSK. At an error rate of $10^{-2}$, the PPM system requires approximately $2 \mathrm{~dB}$ less power than the PSK based system. 


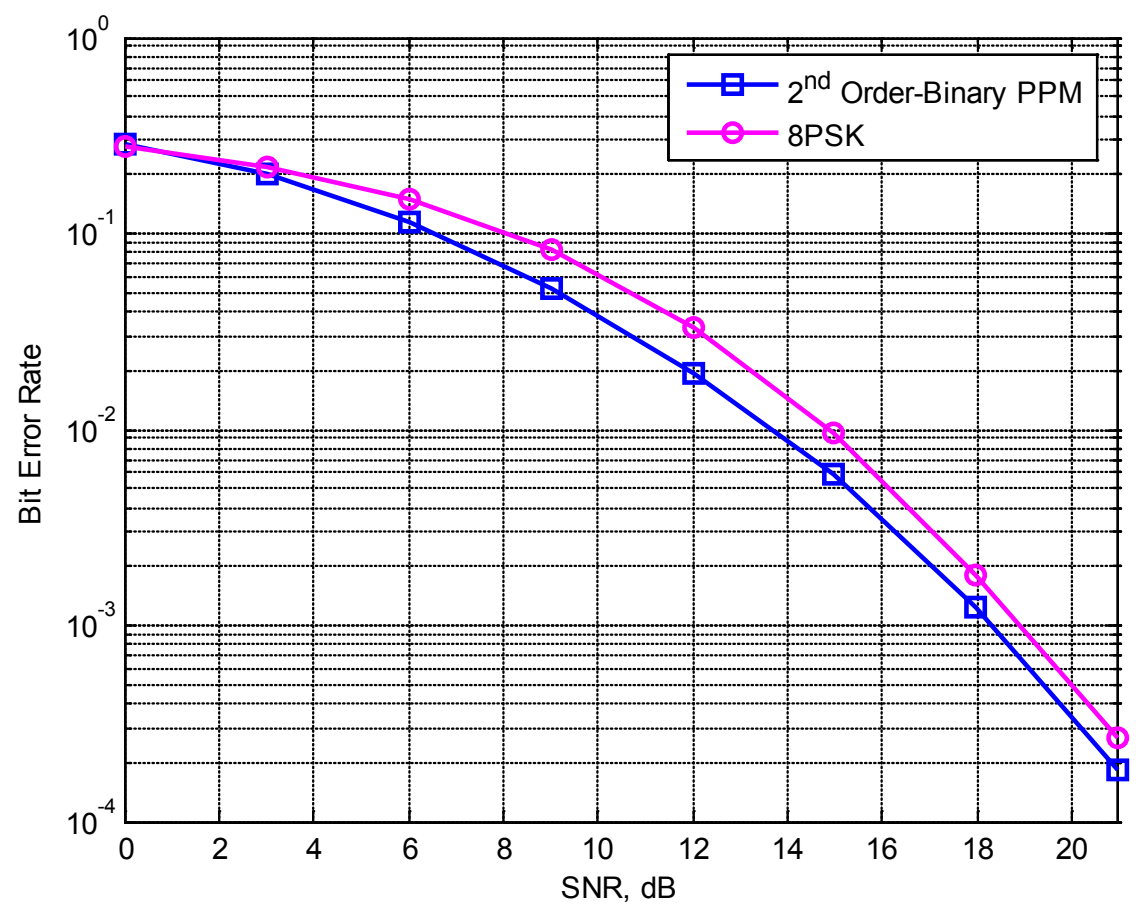

Fig. 4.9 BER Performance for QOSTBC using Full Diversity 8PSK and $2^{\text {nd }}$ Order-Binary PPM

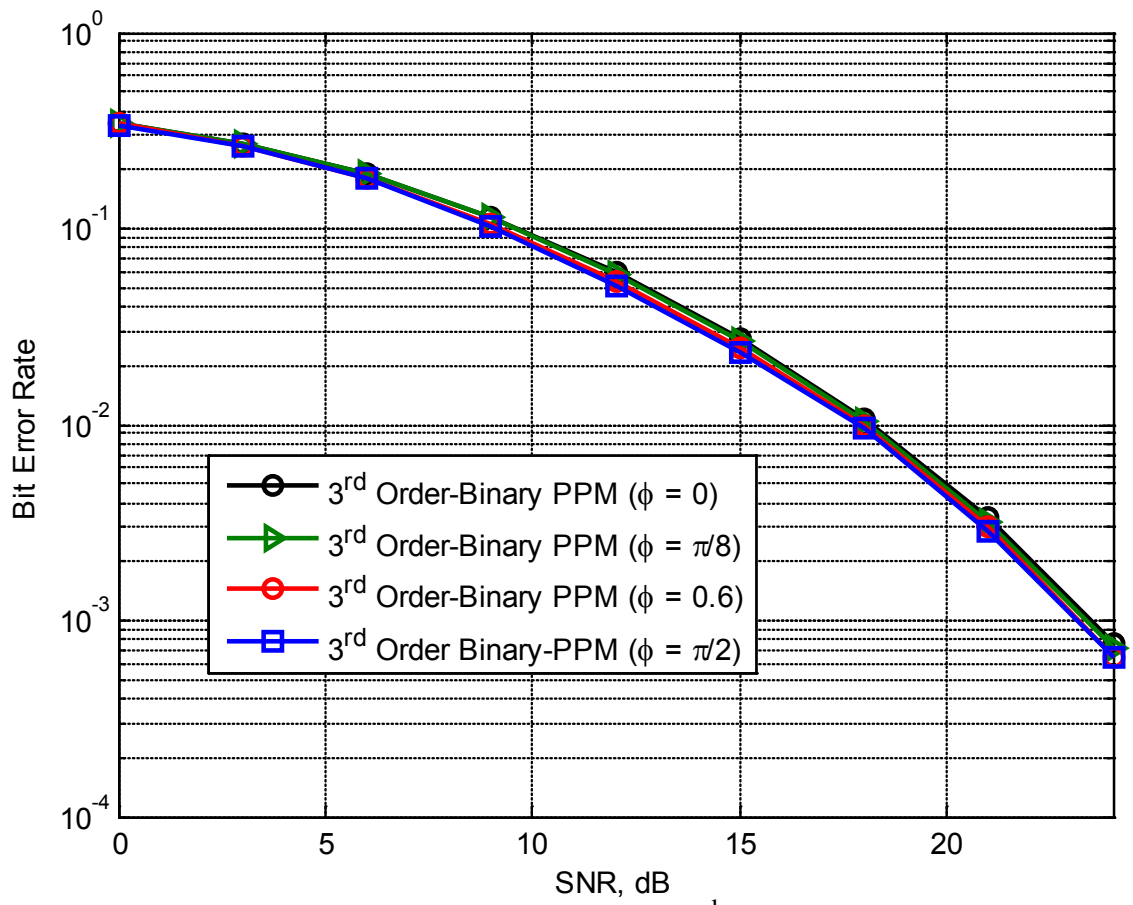

Fig. 4.10 BER Performance for QOSTBC using $3^{\text {rd }}$ Order-Binary PPM for Different Rotation Angles 
Table 4.2 BER Performance for QOSTBC using $3^{\text {rd }}$ Order Binary-PPM for Different Rotation Angles

\begin{tabular}{|c|c|c|c|c|}
\hline & \multicolumn{4}{|c|}{ BER for 3 ${ }^{\text {rd }}$ Order Binary-PPM } \\
\hline SNR & $\phi=\pi / 2$ & $\phi=0.6$ & $\phi=\pi / 8$ & $\phi=0$ \\
\hline 0 & $3.390 \mathrm{E}-01$ & $3.417 \mathrm{E}-01$ & $3.442 \mathrm{E}-01$ & $3.439 \mathrm{E}-01$ \\
\hline 3 & $2.636 \mathrm{E}-01$ & $2.672 \mathrm{E}-01$ & $2.724 \mathrm{E}-01$ & $2.732 \mathrm{E}-01$ \\
\hline 6 & $1.788 \mathrm{E}-01$ & $1.828 \mathrm{E}-01$ & $1.894 \mathrm{E}-01$ & $1.913 \mathrm{E}-01$ \\
\hline 9 & $1.026 \mathrm{E}-01$ & $1.053 \mathrm{E}-01$ & $1.129 \mathrm{E}-01$ & $1.146 \mathrm{E}-01$ \\
\hline 12 & $5.159 \mathrm{E}-02$ & $5.324 \mathrm{E}-02$ & $5.857 \mathrm{E}-02$ & $5.932 \mathrm{E}-02$ \\
\hline 15 & $2.350 \mathrm{E}-02$ & $2.446 \mathrm{E}-02$ & $2.647 \mathrm{E}-02$ & $2.721 \mathrm{E}-02$ \\
\hline 18 & $9.582 \mathrm{E}-03$ & $9.760 \mathrm{E}-03$ & $1.035 \mathrm{E}-02$ & $1.070 \mathrm{E}-02$ \\
\hline 21 & $2.844 \mathrm{E}-03$ & $2.990 \mathrm{E}-03$ & $3.142 \mathrm{E}-03$ & $3.399 \mathrm{E}-03$ \\
\hline 24 & $6.525 \mathrm{E}-04$ & $6.575 \mathrm{E}-04$ & $7.300 \mathrm{E}-04$ & $7.717 \mathrm{E}-04$ \\
\hline
\end{tabular}

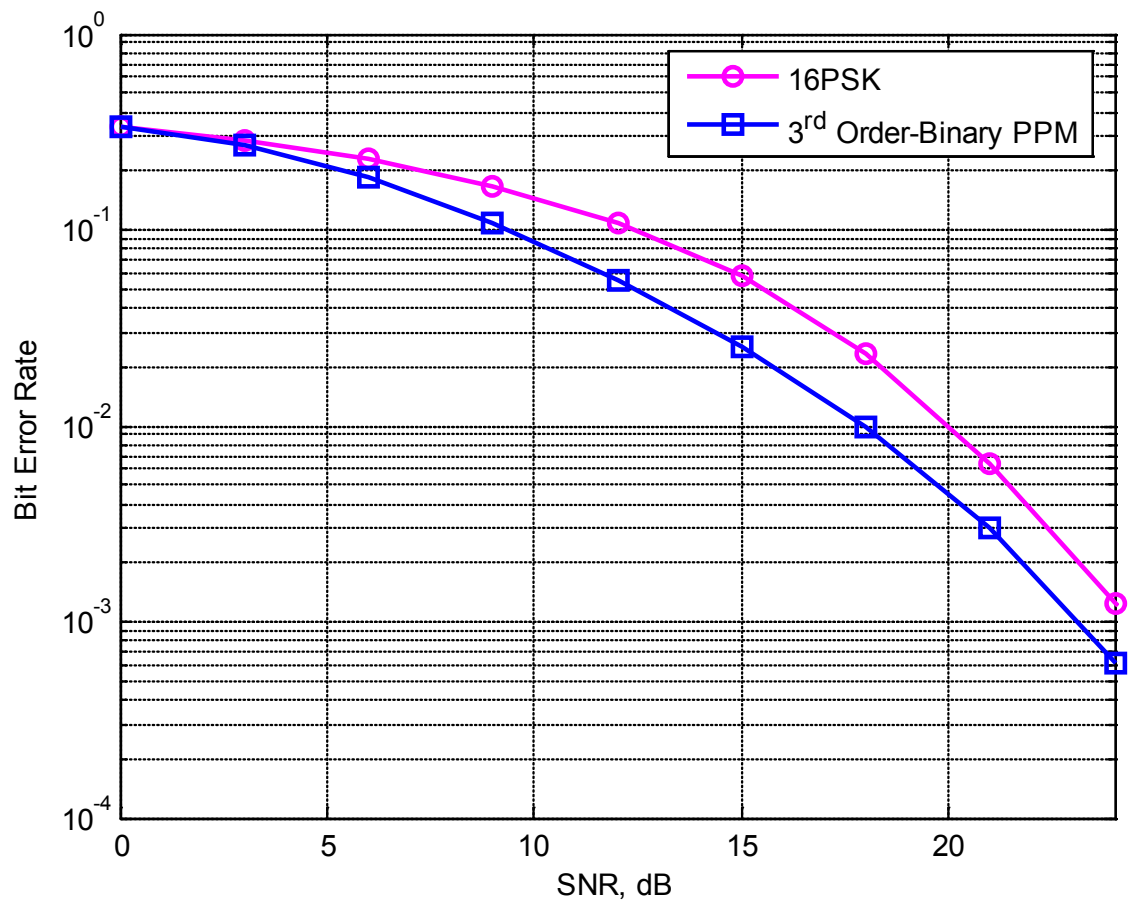

Fig. 4.11 BER Performance for QOSTBC using Full Diversity 16PSK and $3^{\text {rd }}$ OrderBinary PPM 


\subsection{Conclusions}

In this chapter, it was proposed to make use of PPM modulated signals and QOSTBCs with the aim of improving the BER performance and thus, the power efficiency of multiple antenna systems where four antennas are used at the transmitter end. However, in order to take advantage of the multi-path characteristics of the channel, full-diversity must be ensured. Also, it was noted that the highest diversity order can only be achieved by selecting half of the symbols from an optimally rotated constellation. Therefore, the optimal rotation angles for binary linear and quadratic PPM were obtained by maximizing the system's $C G D_{\min }$ and maximizing the $C I C_{\max }$. Here, we found that binary PPM signals of any order achieve close to optimal performance when the rotation angle is $\pi / 2$. Then, through Monte Carlo simulations it was shown that by using the optimal rotation angle, high diversity order and thus an improved BER performance at high SNRs can be attained. In these simulations it was demonstrated that the best BER performance for the PPM-QOSTBC systems is obtained using the proposed selection criteria for the rotation angle based on the $C G D_{\min }$ and $C I C_{\max }$. In addition, it was demonstrated that the proposed full diversity PPM-QOSTBC systems outperform systems using conventional PSK constellations in terms of their error rate, especially at low SNR. 


\section{CHAPTER 5}

\section{SYMBOL-WISE DECODING FOR QOSTBC ENCODED PPM SIGNALS}

The increase in antennas at the transmitter can bring significant improvements in error rate performance if the channels from each antenna are uncorrelated. However, for systems using complex constellations and more than two transmit antennas this also implies an increase in decoding complexity when an optimal maximum likelihood decoder is used. This is because in such cases only pairwise decoding is possible. The decoding complexity of space-time coded systems for more than two transmit antennas can be reduced by using suboptimal decoding techniques such as the quasi-zero forcing (ZF) method proposed by Jeong in [15]. Here, the received vector is decoupled to allow for symbol-wise decoding. This method effectively leads to lower decoding complexity at the expense of a decrease in error rate performance. In [15], however, the decoupling matrix was only derived for the ABBA-QOSTBC proposed by Tirkkonen, Boariu, and Hottinen and the performance of the system was studied only for conventional constellations such as QAM.

In this chapter, a decoupling matrix for the Jafarkhani code is obtained. The decoupling matrix is used to perform symbol-wise decoding on PPM-QOSTBC systems using the quasi-ZF method from [15]. Then, through Monte Carlo simulations, we evaluate and compare the error rate performance of these systems when a maximum likelihood (ML), a sub-optimal maximum likelihood (S-ML), and a high-order ambiguity function (HAF) based decoders are used for demodulation. 


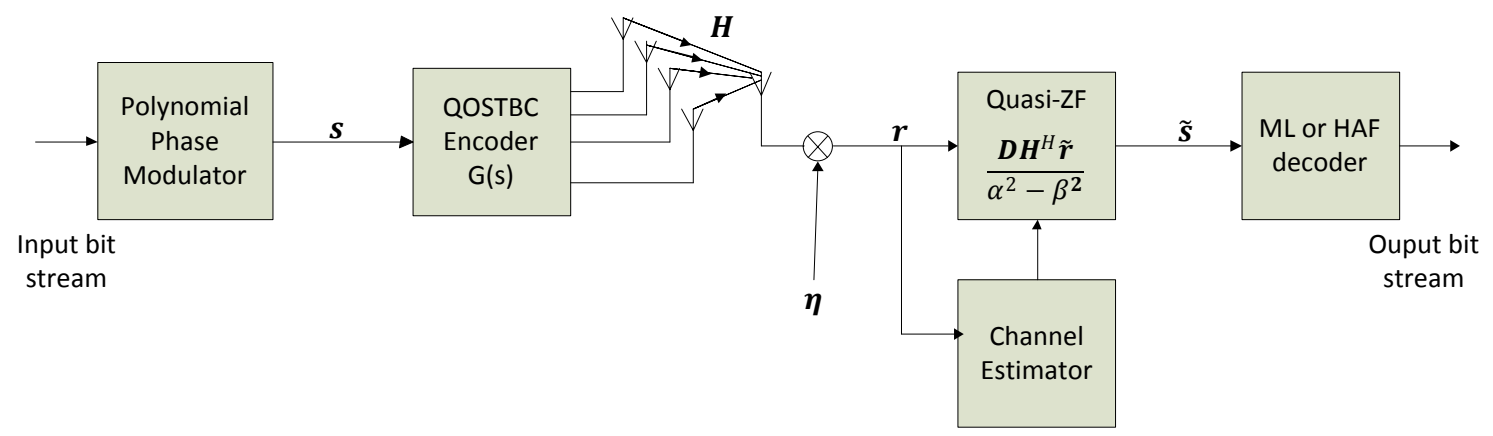

Fig. 5.1. Transmitter and Receiver Model for PPM-QOSTBC using Quasi-ZF

\subsection{System Model}

The system under study from now on will be referred to as PPM-QOSTBC and its model is shown in Fig. 5.1. The specific QOSTBC employed in this chapter is that proposed by Jafarkhani in [4]. Then, given a set of PPM signals described as:

$$
s(t)=\exp \left(j\left(\omega_{c} t+\varphi(t)\right)\right.
$$

for $(k-1) T \leq t \leq k T$ and $k=\{1,2,3,4\}$ (four symbol intervals). The transmission matrix is:

$$
\boldsymbol{G}(s)=\left(\begin{array}{rrrr}
s_{1}(t) & s_{2}(t) & s_{3}(t) & s_{4}(t) \\
-s_{2}^{*}(t) & s_{1}^{*}(t) & -s_{4}^{*}(t) & s_{3}^{*}(t) \\
-s_{3}^{*}(t) & -s_{4}^{*}(t) & s_{1}^{*}(t) & s_{2}^{*}(t) \\
s_{4}(t) & -s_{3}(t) & -s_{2}(t) & s_{1}(t)
\end{array}\right)
$$

The received signal $r_{k}$ at symbol interval $k$ is then given by

$$
r_{k}(t)=\sum_{n=1}^{4} \alpha_{n} e^{j \theta_{n}} G(s)_{k, n}+\eta_{k}(t)
$$


As it can be seen from the code in (5.2), four symbols are transmitted in four symbol intervals. This indicates that this is in fact a full rate code; however, because the columns of matrix $\boldsymbol{G}(s)$ are not orthogonal to each other, only pairwise ML decoding can be performed at the receiver. This obviously leads to a higher decoding complexity than that of OSTBCs. To address this issue one could use a zero-forcing (ZF) algorithm described as [14]:

$$
\tilde{\boldsymbol{s}}=\left(\boldsymbol{H}^{H} \boldsymbol{H}\right)^{-\mathbf{1}} \boldsymbol{H}^{H} \tilde{\boldsymbol{r}}
$$

This is a suboptimal technique commonly used to enable symbol-wise decoding and reduce the complexity of MIMO systems. Nevertheless, this method still involves highly complex operations such as the computation of a matrix inverse. Therefore, instead of zero-forcing, we apply the quasi-ZF scheme proposed in [15] to the Jafarkhani code.

For convenience, the channel coefficients are denoted as $h_{n}=\alpha_{n} e^{j \theta_{n}}$ for $1 \leq n \leq 4$. Again, it is assumed that these coefficients are constant for one transmission block and change independently from one block to the next. Then, to apply the aforementioned quasi-ZF method, we take the complex conjugate of the second and third components of the received signal in (5.3). After writing the resulting expression in vector notation, we obtain

$$
\tilde{\boldsymbol{r}}=\boldsymbol{H} \boldsymbol{s}+\widetilde{\boldsymbol{\eta}}
$$

where the transformed received signal vector is given as $\tilde{\boldsymbol{r}}=$ $\left[r_{1}(t), r_{2}{ }^{*}(t), r_{3}{ }^{*}(t), r_{4}(t)\right]^{T}, \quad$ the transmitted sequence vector is $\boldsymbol{s}=\left[s_{1}(t), s_{2}(t), s_{3}(t), s_{4}(t)\right]^{T}, \widetilde{\boldsymbol{\eta}}=\left[\eta_{1}(t), \eta_{2}{ }^{*}(t), \eta_{3}{ }^{*}(t), \eta_{4}(t)\right]^{T},(\boldsymbol{\square})^{T}$ denotes the transpose operation, and $\boldsymbol{H}$ is the channel matrix given by 


$$
\boldsymbol{H}=\left(\begin{array}{cccc}
h_{1} & h_{2} & h_{3} & h_{4} \\
h_{2}^{*} & -h_{1}^{*} & h_{4}^{*} & -h_{3}^{*} \\
h_{3}^{*} & h_{4}^{*} & -h_{1}^{*} & -h_{2}^{*} \\
h_{4} & -h_{3} & -h_{2} & h_{1}
\end{array}\right)
$$

Then,

$$
\boldsymbol{H}^{H} \boldsymbol{H}=\left(\begin{array}{cccc}
\alpha & 0 & 0 & \beta \\
0 & \alpha & -\beta & 0 \\
0 & -\beta & \alpha & 0 \\
\beta & 0 & 0 & \alpha
\end{array}\right)
$$

where $(\boldsymbol{\square})^{H}$ is the complex conjugate transpose, $\alpha=\left|h_{1}^{2}\right|+\left|h_{2}^{2}\right|+\left|h_{3}^{2}\right|+\left|h_{4}^{2}\right|$, and $\beta=2 \operatorname{Re}\left(h_{1} h_{4}^{*}-h_{2} h_{3}^{*}\right)$.

The product of the transformed received sequence and the Hermitian of the channel matrix is given as:

$$
\boldsymbol{H}^{H} \tilde{\boldsymbol{r}}=\boldsymbol{H}^{H} \boldsymbol{H} \boldsymbol{s}+\boldsymbol{H}^{H} \widetilde{\boldsymbol{\eta}}
$$

By finding a decoupling matrix $\boldsymbol{D}$ such that the interference components, $\beta$, of the product $\boldsymbol{D} \boldsymbol{H}^{H} \boldsymbol{H}$, are forced to zero, symbol-wise estimation of the transmitted sequences can be performed. Based on this and from the Gramian matrix in (5.7), the decoupling matrix $\boldsymbol{D}$ for the Jafarkhani scheme is obtained as

$$
\boldsymbol{D}=\left(\begin{array}{cccc}
\alpha & 0 & 0 & -\beta \\
0 & \alpha & \beta & 0 \\
0 & \beta & \alpha & 0 \\
-\beta & 0 & 0 & \alpha
\end{array}\right)
$$

To obtain this matrix all is needed is to multiply the $\beta$ parameters by -1 . Notice that this process was also independently described in [23]. Then using (5.5), (5.7), and (5.9), the transmitted sequence estimates are given as 


$$
\tilde{\boldsymbol{s}}=\frac{\boldsymbol{D} \boldsymbol{H}^{H} \tilde{\boldsymbol{r}}}{\alpha^{2}-\beta^{2}}
$$

where $\tilde{\boldsymbol{s}}=\left[\tilde{s}_{1}(t), \tilde{s}_{2}(t), \tilde{s}_{3}(t), \tilde{s}_{4}(t)\right]^{T}$. At this point, we can observe that the signals in $\tilde{\boldsymbol{s}}$ are PPM signals embedded in noise and can thus be demodulated through the ML, S-ML or HAF algorithms presented in chapter 2.

\subsection{Simulation Results}

In the following Monte Carlo simulations, the BER performance of a 4x1 QOSTBC system using quasi- ZF for PPM and PSK modulations of various orders is studied. For these simulations it is assumed that the information is transmitted over a quasi-static frequency flat Rayleigh fading channel and that the channel state information (CSI) is perfectly known at the receiver. In addition to this, Gray bit labeling has been applied to the PPM and PSK symbols used in the simulations. In Fig. 5.2, we compare the performance of linear-binary PPM-QOSTBC and QPSK-QOSTBC. Here, it can be observed that because of the similarity in the Euclidean distances of both modulation schemes, the proposed linear-binary PPM system has the same error rate performance as the system using QPSK. Furthermore, in the same figure, it is shown that the error rates of the linear-binary PPM-QOSTBC systems using S-ML and HAF are almost the same and clearly suboptimal when compared to the system using ML. In Fig. 5.3, we also consider the error rate performance of $2^{\text {nd }}$ order-binary PPM-QOSTBC system using ML, S-ML, and HAF decoding structures and compare them to that of an 8PSK-QOSTBC system. The $2^{\text {nd }}$ order binary-PPM-based systems' performance is clearly better than that of the QOSTBC using 8PSK. Moreover, the system using the ML decoder has a lower error rate than the PPM-QOSTBC system using HAF as its decoding technique. 


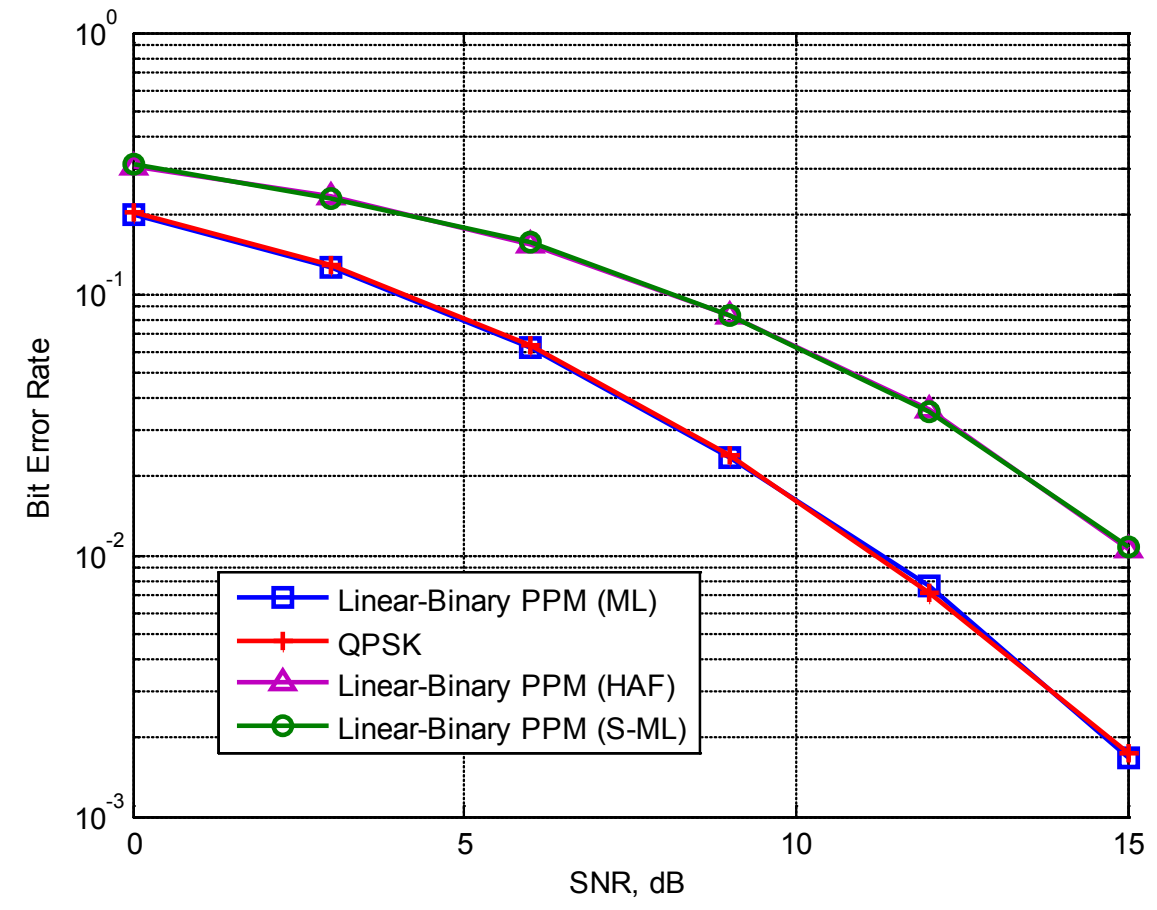

Fig. 5.2. BER Performance Comparison of Linear-Binary PPM-QOSTBC and QPSKQOSTBC using Quasi-ZF

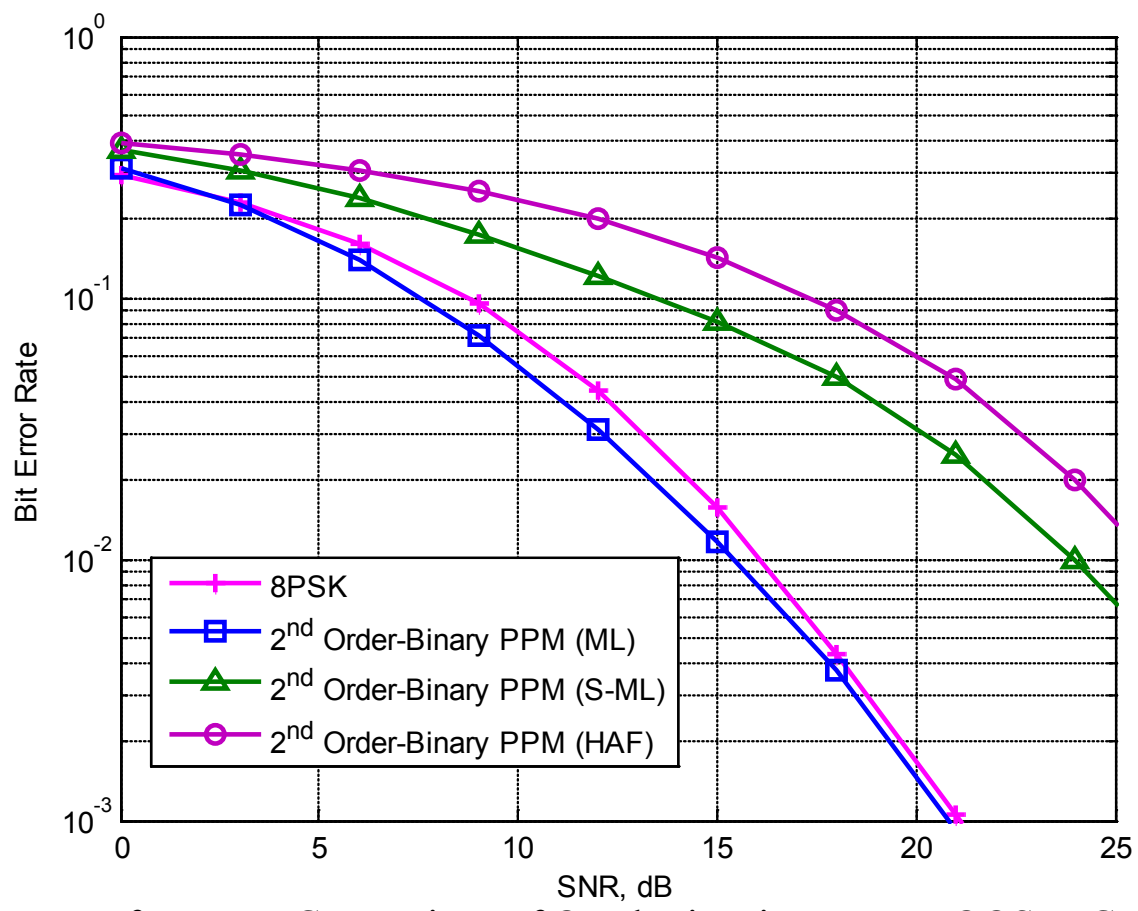

Fig. 5.3. BER Performance Comparison of Quadratic-Binary PPM-QOSTBC and 8PSKQOSTBC using Quasi-ZF 
Finally, in Fig. 5.4 the error rate curves for a 3rd order-binary PPM-QOSTBC and a 16PSK-QOSTBC system are displayed. A lower BER is again clearly observed for the PPM-based system. In particular, at a BER of $10^{-1}$ the proposed scheme requires $5 \mathrm{~dB}$ less energy to transmit a symbol than its PSK-based counterpart.

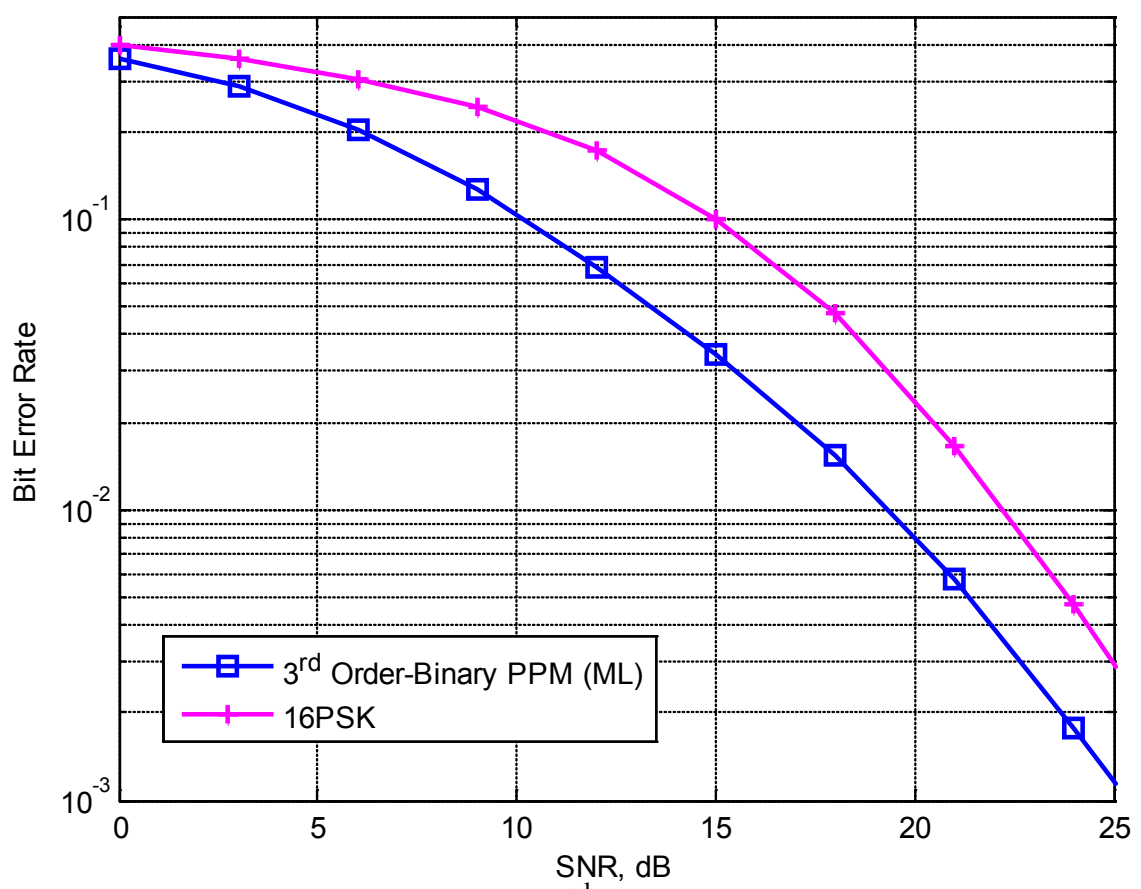

Fig. 5.4. BER Performance Comparison of $3^{\text {rd }}$ Order-Binary PPM-QOSTBC and 16PSKQOSTBC using Quasi-ZF

\subsection{Conclusions}

In this chapter, we investigated the use of symbol-wise decoding for polynomial phase modulated (PPM) signals in wireless communication systems with four transmit antennas. In this specific case, we studied the performance of a QOSTBC, proposed by Jafarkhani, with PPM signals being used as the modulation format. To enable symbol- 
wise decoding, we obtained a decoupling matrix, specifically for the Jafarkhani scheme, and applied a quasi zero-forcing technique to eliminate interference between symbols. Decoding was again performed using three different methods ML, S-ML, and HAF. We compared the proposed systems to QOSTBC systems using PSK constellations, and simulation results showed that the proposed QOSTBC systems outperform, in terms of BER performance, the zero-forced QOSTBC systems using PSK. The lower error rates for PPM-based systems stem from the high order coefficients robustness to the effects of fading and the fact that when decoding PPM signals their Euclidean distance between any pair of possible symbols, which is in general higher than that of PSK constellations, yields a lower probability of error than for PSK-based systems. This shows that the proposed systems have potential to be used in power efficient communication systems as lower power is required to transmit information at a given error rate. Lastly, it was also observed that the PPM systems using ML decoding had a considerable improvement in performance when compared to the other systems at the expense of higher complexity. 


\section{CHAPTER 6}

\section{CONCLUSIONS AND FUTURE WORK}

In this dissertation, the bit-error-rate (BER) performance of different orders of polynomial phase modulated (PPM) signals for single-input single-output (SISO) systems were evaluated when three different types of decoders were employed and gray bit labelling was applied to the modulated symbols. It was shown that PPM SISO systems outperform systems using conventional modulations such as PSK especially at high transmission rates.

Multiple antenna systems using PPM signals were also proposed in order to take advantage of their lower error rates. For systems with two transmit antennas, the BER of different PPM OSTBC schemes was evaluated and compared to that of PSK OSTBC systems. As in the SISO case, different decoder structures for PPM signals were evaluated. As expected when an optimum ML decoder is employed, the PPM based systems outperformed those using PSK. For systems with four transmit antennas, PPM signals were encoded using the Jafarkhani QOSTBC in order to take full advantage of the multipath channel. However, to achieve the highest diversity order, a new selection criterion known as the maximum channel interference coefficient (CIC) was introduced. The optimal rotation angles for different PPM signal orders were obtained by selecting the values that would maximize the minimum coding gain distance (CGD) and minimize the maximum CIC for each respective system. Using Monte Carlo simulations it was shown that the angles obtained through this method provide the best error rate performance for the given PPM systems. That is, for a required error rate, the proposed 
full-diversity PPM QOSTBC systems require less transmission power per symbol than full-diversity PSK QOSTBC and PPM QOSTBC systems without full diversity. Therefore, the resulting systems were shown to have higher transmission power efficiency ultimately leading to larger battery life for mobile wireless devices.

Lastly, PPM QOSTBC systems using symbol-wise decoding were proposed in order to reduce the decoding complexity of a PPM system with four transmit antennas. This decrease in system complexity was achieved by using a quasi zero-forcing technique along with the Jafarkhani QOSTBC. Contrary to full diversity PPM-QOSTBC systems, where only pairwise ML decoding is possible, the resulting systems can be used along with simple decoding structures such as S-ML and HAF as they can be applied on a symbol by symbol basis. It is important to note that, as shown in the simulation results, this reduction in complexity comes at the expense of a lower error rate performance.

In general, it was demonstrated that PPM-based systems provide an attractive alternative to conventional modulation formats in cases such as mobile wireless devices where transmit power efficiency is an issue. As future work one might consider the further study of the BER performance and the spectral efficiency of the PPM-QOSTBC system. Finding a closed form or an approximate invertible expression for these two parameters is crucial for the implementation of adaptive rate and power allocation algorithms for multiple-input multiple-output systems [17]. In such case, the system's transmission rate and power at each antenna can be adapted based on the channel condition.

Finally, by setting the constant component of a PPM signal as a storage variable for the phase of the previously transmitted signal, a constant envelope PPM can be generated [21]. This constant envelope signal can be coupled with a STBC in order to further 
reduce the system's peak-to-average power ratio (PAPR). The issue of reducing a system's PAPR is of particular relevance in orthogonal frequency division multiplexing (OFDM) systems. High PAPR values increase the amount of quantization errors in the analog-to-digital and digital-to-analog conversion processes and, perhaps more importantly, increase the system's sensitivity to the power amplifier nonlinearities [24]. 


\section{REFERENCES}

[1] M. Deruyck; E. Tanghe; W. Joseph; W. Vereecken; M. Pickavet; B. Dhoedt; L. Martens; , "Towards a deployment tool for wireless access networks with minimal power consumption," IEEE 21st International Symposium on Personal, Indoor and Mobile Radio Communications Workshops (PIMRC Workshops), 2010, vol., no., pp.295-300, 26-30 Sept. 2010

[2] Lizhong Zheng; Tse, D.N.C.; "Diversity and multiplexing: a fundamental tradeoff in multiple-antenna channels," IEEE Transactions on Information Theory, vol.49, no.5, pp. 1073- 1096, May 2003

[3] S. M. Alamouti, "A simple transmitter diversity scheme for wireless communications," IEEE J. Select. Areas Commun., vol. 16, pp.1451-1458, Oct. 1998.

[4] H. Jafarkhani, "A quasi-orthogonal space-time block code," IEEE Transactions on Communications, vol.49, no.1, pp.1-4, Jan 2001.

[5] W. Su and X.G. Xia, "Signal constellations for quasi-orthogonal space-time block codes with full diversity," IEEE Trans. Inf. Theory, vol.50, no.10, pp.2331-2347, Oct. 2004.

[6] Chiang-Yu Chen; Ming-Yang Chen; Cioffi, J.M.; , "Full-diversity quasi-orthogonal space-time block codes for M-PSK modulations," Global Telecommunications Conference, 2005. GLOBECOM '05. IEEE, vol.5, no., pp.5 pp.-3026, 2-2 Dec. 2005

[7] Dong Wang; Xiang-Gen Xia; , "Optimal diversity product rotations for quasiorthogonal STBC with MPSK symbols," Communications Letters, IEEE , vol.9, no.5, pp. 420- 422, May 2005

[8] R. Sinha; G. E. Atkin; C. Zhou, "Performance Evaluation of Variable-Rate Polynomial Phase Modulation," Military Communications Conference, 2007. MILCOM 2007. IEEE, vol., no., pp.1-7, 29-31 Oct. 2007.

[9] H.P. Dam; Lun Huang; G.E. Atkin, "Mimo/-space time codes for Polynomial Phase modulation wireless communications," Military Communications Conference, 2009. MILCOM 2009. IEEE, vol., no., pp.1-7, 18-21 Oct. 2009.

[10] S. Barbarossa; A. Scaglione, "Demodulation of CPM signals using piecewise polynomial-phase modeling," Proceedings of the 1998 IEEE International Conference on Acoustics, Speech and Signal Processing, 1998, vol.6, no., pp.32813284 vol.6, 12-15 May 1998. 
[11] Y. Jianming; G.B. Giannakis, "Product multi-lag high-order ambiguity function for blind equalization of polynomial phase signals," Proceedings of the IEEE-SP International Symposium on Time-Frequency and Time-Scale Analysis, 1996, vol., no., pp.529-532, 18-21 Jun 1996

[12] H. Jafarkhani, Space-Time Coding: Theory and Practice, Cambridge University Press, 2005.

[13] O. Tirkkonen; A. Boariu; A. Hottinen; , "Minimal non-orthogonality rate 1 spacetime block code for 3+ Tx antennas," 2000 IEEE Sixth International Symposium on Spread Spectrum Techniques and Applications, vol.2, no., pp.429-432 vol.2, 2000.

[14] C.B. Papadias; G.J. Foschini; , "Capacity-approaching space-time codes for systems employing four transmitter antennas," IEEE Transactions on Information Theory, vol.49, no.3, pp. 726- 732, Mar 2003.

[15] Jae-Sun Jeong; Su-Bin Park; Chang-Bum Jo; Eun-Ji Yoo; Youn-Shik Byun; , "Quasi-orthogonal space-time block codes based on single symbol decoding for four transmit antennas," 11th International Conference on Advanced Communication Technology, 2009. ICACT 2009, vol.03, no., pp.1742-1745, 15-18 Feb. 2009.

[16] B. Boashash; , "Estimating and interpreting the instantaneous frequency of a signal. II. Algorithms and applications,"Proceedings of the IEEE, vol.80, no.4, pp.540568, Apr 1992

[17] Zhendong Zhou; B. Vucetic; M. Dohler; Yonghui Li, "MIMO systems with adaptive modulation," IEEE Transactions on Vehicular Technology, vol.54, no.5, pp. 1828-1842, Sept. 2005.

[18] J.G. Proakis, Digital communications. 4th ed. McGraw-Hill series in electrical and computer engineering. 2001, Boston ; London: McGraw-Hill, xxi, 1002.

[19] S. Peleg; B. Friedlander; "The discrete polynomial-phase transform," IEEE Transactions on Signal Processing, vol.43, no.8, pp.1901-1914, Aug 1995.

[20] V. Tarokh; N. Seshadri; A.R. Calderbank; , "Space-time codes for high data rate wireless communication: performance criterion and code construction," IEEE Transactions on Information Theory, vol.44, no.2, pp.744-765, Mar 1998

[21] R. Sinha, "Digital Phase Modulation Using Polynomial Phase Modulated Signals," in Ph.D Dissertation, Electrical and Computer Engineering. Chicago: Illinois Institute of Technology, May 2007. 
[22] E. Agrell; Johan Lassing; E.G. Strom; T. Ottosson; , "Gray Coding for Multilevel Constellations in Gaussian Noise," IEEE Transactions on Information Theory, , vol.53, no.1, pp.224-235, Jan. 2007

[23] Pham Van Bien; Weixing Sheng; Xiaofeng Ma; Hao Wang; , "Improved decoder schemes for QOSTBCs based on single-symbol decoding," Advanced Technologies for Communications (ATC), 2010 International Conference on , vol., no., pp.7-10, 20-22 Oct. 2010.

[24] Xiaowen Gu; Seungmin Baek; Suwon Park; , "PAPR reduction of OFDM signal using an efficient SLM technique," The 12th International Conference on Advanced Communication Technology (ICACT), 2010, vol.1, no., pp.324-328, 7-10 Feb. 2010. 
VITA

\section{OMAR GRANADOS}

2005

B.S., Electrical Engineering, Florida International University, Miami, FL.

2011

Doctoral Candidate, Electrical Engineering

Florida International University

Miami, FL

\section{PUBLICATIONS AND PRESENTATIONS}

Journal

O. Granados, J. Andrian, "Quasi-Orthogonal Space Time Block Coding using Polynomial Phase Modulation" Submitted to ISRN Communications and Networking Journal

Conference

O. Granados, J. Andrian, "Space-Time Block Coding with Symbol-Wise Decoding for Polynomial Phase Modulated Signals" IEEE Wireless Telecommunications

Symposium (WTS 2011), New York, New York. 2011

R. Duarte, O. Granados, C. Liu, J. Andrian, “Case Study on a Software Communications Architecture Component for Hardware Acceleration” Submitted to The 4th International Conference on Ubi-media Computing (U-Media 2011), Sao Paulo, Brazil. 2011

H. Qiang, O. Granados, J. Andrian, J. Luo, M. Woolley, "Implementation of a WLAN Waveform under Cost-Effective Novel Software and Hardware Architecture" SDR Forum, 2008

Lectures

"Introduction to Software Defined Radio" Invited Lecture

Student Colloquium, Delaware State University, October 30, 2008.

\section{OTHER ACTIVITIES}

Instructor: Signals and Systems, Electronics I, Systems Laboratory, Logic Design Laboratory 
Teaching Assistant: Control Systems I, Fields and Waves, Biomedical Instrumentation Student Member, Institute of Electrical and Electronics Engineers (IEEE)

Co-Author, "Extensible Affordable Software Defined Radio with Cross Band and Cross Protocol Capabilities" (Funded: Phase I)

Project: Design and Development of Cognitive Jamming (Funded by Air Force Research $\mathrm{Lab}$ 